\title{
Isolated and combined genetic tremor syndromes: a critical appraisal based on the 2018 MDS criteria
}

\author{
Francesca Magrinellia, ${ }^{a, b}$ Anna Latorre ${ }^{a}$, Bettina Balint ${ }^{\text {a,c }}$, Melissa Mackenzie ${ }^{\mathrm{a}}$, Eoin Mulroy ${ }^{\mathrm{a}}$, \\ Maria Stamelou ${ }^{d}$, Michele Tinazzi ${ }^{b}$, Kailash P. Bhatia ${ }^{a^{*}}$
}

${ }^{a}$ Department of Clinical and Movement Neurosciences, UCL Queen Square Institute of Neurology, London, United Kingdom [f.magrinelli@ucl.ac.uk, a.latorre@ucl.ac.uk, b.balint@ucl.ac.uk, melissa.mackenzie@alum.utoronto.ca, eoin.mulroy@nhs.net]

${ }^{\mathrm{b}}$ Department of Neurosciences, Biomedicine and Movement Sciences, University of Verona, Verona, Italy [michele.tinazzi@univr.it]

${ }^{\mathrm{c}}$ Department of Neurology, University Hospital Heidelberg, Heidelberg, Germany

d Department of Neurology, Attikon University Hospital, Athens, Greece [mariastamelou@gmail.com]

* Corresponding author

Professor Kailash P. Bhatia

Department of Clinical and Movement Neurosciences, UCL Queen Square Institute of Neurology

Queen Square, London WC1N 3BG, United Kingdom

E-mail: k.bhatia@ucl.ac.uk

\author{
Word count \\ Abstract: 250 \\ Main text: 4,384 \\ Tables: 5 \\ Figure: 1 \\ Video: 1 \\ Supplementary File: 1 \\ References: 164
}

\section{Key words}

Tremor; essential tremor; genetics; movement disorders; diagnosis 


\begin{abstract}
The 2018 consensus statement on the classification of tremors proposes a two-axis categorization scheme based on clinical features and etiology. It also defines "isolated" and "combined" tremor syndromes depending on whether tremor is the sole clinical manifestation or is associated with other neurological or systemic signs. This syndromic approach provides a guide to investigate the underlying etiology of tremors, either genetic or acquired.

Several genetic defects have been proven to cause tremor disorders, including autosomal dominant and recessive, X-linked, and mitochondrial diseases, as well as chromosomal abnormalities. Furthermore, some tremor syndromes are recognized in individuals with a positive family history, but their genetic confirmation is pending. Although most genetic tremor disorders show a combined clinical picture, there are some distinctive conditions in which tremor may precede the appearance of other neurological signs by years or remain the prominent manifestation throughout the disease course, previously leading to misdiagnosis as essential tremor (ET). Advances in the knowledge of genetically determined tremors may have been hampered by the inclusion of heterogeneous entities in previous studies on ET. The recent classification of tremors therefore aims to provide more consistent clinical data for deconstructing the genetic basis of tremor syndromes in the next-generation and long-read sequencing era.

This review outlines the wide spectrum of tremor disorders with defined or presumed genetic etiology, both isolated and combined, unraveling diagnostic clues of these conditions and focusing mainly on ET-like phenotypes. Furthermore, we suggest a phenotype-to-genotype algorithm to support clinicians in identifying tremor syndromes and guiding genetic investigations.
\end{abstract}




\section{INTRODUCTION}

The 2018 consensus statement on tremor classification reiterates that tremor is an involuntary, rhythmic, oscillatory movement of a body part,[1,2] and provides a novel categorization scheme based on clinical features (Axis 1) and etiology (Axis 2).[1] Within Axis 1, tremor syndromes are termed "isolated" and "combined" depending on whether tremor occurs alone or is associated with other neurological or relevant systemic signs.[1] This syndromic approach aims to better delineate tremor phenotypes and investigations of both genetic and acquired tremor syndromes.[1]

Many tremor disorders have a known genetic basis, including autosomal dominant (AD) and recessive (AR), $\mathrm{X}$-linked and mitochondrial diseases, as well as chromosomal abnormalities. Moreover, some patients with tremor have positive family histories, but lack molecular diagnosis,[1] suggesting Mendelian inheritance of hitherto unknown genes or more complex heritability patterns. Most genetic tremor disorders are combined syndromes. However, in some distinctive conditions, tremor may precede other neurological signs by years or remain the sole or prominent manifestation, potentially masquerading as essential tremor (ET). This review outlines the broad spectrum of isolated and combined tremor syndromes with defined or presumed genetic etiology. Given the numerous genes and chromosomal abnormalities linked to tremor disorders, we focus on ET-like phenotypes and highlight clues to diagnose other distinctive phenotypes. Monogenic disorders are presented first according to their inheritance patterns and chromosomal aberrations thereafter. A phenotype-to-genotype algorithm is provided at the end. Previously unpublished videos (Video 1) and references to previously published videos (Supplementary Table) showing some tremor disorders covered by this review are provided.

\section{INHERITED AUTOSOMAL DOMINANT TREMOR DISORDERS}

\subsection{Essential tremor}

ET is an isolated tremor syndrome characterized by bibrachial action tremor with or without tremor in other body parts (e.g., head, larynx, lower limbs).[1] Prevalence is estimated at $1 \%$ in the general population and 4$5 \%$ in people over 65 years.[3] Age of onset ranges from childhood to late adulthood, with a bimodal distribution showing peaks before 24 years of age, and after 46 years of age.[4] Some affected individuals display alcohol sensitivity.[1,2] Positive family history is present in $20-90 \%$ of ET cases, suggesting AD inheritance.[5] Candidate loci and genes associated with ET are listed in Table 1.[6-29] However, no genetic findings have conclusively been replicated, and ET etiology remains controversial. Many factors may have hampered the dissection of genetic underpinnings of ET.[35] First, the longstanding lack of stringent diagnostic criteria and the lack of biomarkers for ET which may have facilitated contamination of ET study cohorts with tremor disorders misdiagnosed with ET (e.g., Klinefelter syndrome (KS), fragile X-related tremor/ataxia syndrome (FXTAS), DYT-ANO3 (DYT24),[36] and SCA-PPP2R2B (SCA12)[36]), or with 
"ET plus" syndromes, where additional subtle neurological signs (e.g., mild memory impairment, impaired tandem gait, subtle body posturing) are present.[1] Second, the reduced penetrance of ET observed in ET families. Third, the high rate of phenocopies in ET pedigrees, i.e. people with a similar or identical phenotype resulting from different underlying genotypes or environmental nongenetic factors. Fourth, the possible contribution of multiple risk loci, epigenetic modifications, and environmental influences in the pathogenesis of ET.[35] Finally, the intrinsic limitations of genetic technologies available for ET research over time. For instance, early genetic research on ET was mainly represented by genome-wide association studies (GWAS) whose limitations have recently been reviewed by Tam et al.[37] As another example, nextgeneration sequencing (NGS) has not allowed to accurately analyze genomic regions carrying structural variation, repetitive sequences, guanine-cytosine rich sequences, or sequences with multiple homologous elements, but the emerging long-read sequencing (LRS) technologies promise to overcome specific limitations of NGS and might shed light on the significance of noncoding genomic regions, complex genomic variants, repeat expansions, and DNA methylation patterns in the pathogenesis of ET.[38,39] Against this background, LRS has recently contributed to identify a GCC trinucleotide repeat expansion in the NOTCH2NLC gene in 11 Chinese kindreds with ET,[29] but this finding has not been replicated in independent studies yet.

In summary, ET and ET plus represent clinical syndromes of heterogeneous possibly genetic etiology. In this context, the recent classification of tremors should promote deeper clinical phenotyping to deconstruct genetic tremor syndromes in the NGS and LRS era.[1]

\subsection{Autosomal dominant primary dystonias}

Tremor is part of the motor phenomenology of dystonia,[40] therefore all genes associated with dystonia may underpin isolated and combined tremor syndromes.[1] Dystonic tremor manifests as a rhythmic, intermittent, patterned movement in body regions which are primarily affected or unaffected by dystonia. It may appear before overt dystonic posturing and abate in response to sensory tricks or null-point positioning.[40] An overview of monogenic primary dystonias is found elsewhere, including the AD dystonia phenotypes related to TORIA (DYT1), THAP1 (DYT6), and GNAL (DYT25) genes.[41-45] Tremor is often reported as a major clinical feature in DYT-ANO3 and DYT/PARK-GCH1 (DYT5a),[36] discussed below.

DYT-ANO3 is caused by a heterozygous mutation in ANO3, which maps on chromosome 11p14.2-p14.3 and encodes anoctamin 3, a Ca2 ${ }^{+}$-gated chloride channel highly expressed in the striatum.[46] Age of onset ranges between childhood and the sixth decade.[46-48] Tremor involving the head, voice, and upper limbs in various combinations is often the earliest manifestation, and most prominent finding throughout the disease course, mimicking ET or SCGE-related myoclonus-dystonia (Supplementary Table); dystonic posturing is often minimal.[49] Contiguous spread over years is possible, but generalization has never been reported.[49] Subcortical myoclonus can also be observed.[49] 
DYT/PARK- $G C H 1$ is a dopa-responsive dystonia caused by a heterozygous mutation in $G C H 1$, which maps on chromosome 14q22.1-q22.2 and encodes the enzyme GTP cyclohydrolase 1.[50] Upper-limb postural tremor has been reported, either alone or with parkinsonism, in both childhood- and adult-onset cases.[50,51] Voice, head, chin and parkinsonian rest tremor are also described.[51] Tremor is dopa-responsive and shows diurnal fluctuation.[50] AR inheritance has also been reported in DYT/PARK-GCH1.[52]

\subsection{Autosomal dominant spinocerebellar ataxias}

Tremor is common in AD spinocerebellar ataxias (SCAs). A 2-3 Hz titubatory head or trunk tremor reflects cerebellar dysfunction, and is therefore not indicative of a specific SCA. However, action tremor of the hands resembling ET has been reported as presenting sign in SCA-PPP2R2B and SCA-FGF14 (SCA27),[36] which are herein discussed in detail.[53-55] Palatal tremor is part of the phenotypic spectrum of SCA20 and SCA-ATXN7 (SCA7),[36] both important differential diagnoses of progressive ataxia with palatal tremor (PAPT; see below).[56-58] Rest tremor is rare in SCAs, usually indicating coexisting parkinsonism, as observed in SCA-ATXN2 (SCA2) and SCA-ATXN3 (SCA3).[36,59]

SCA-PPP2R2B (SCA12) is caused by a CAG/CTG repeat expansion in the 5 ' untranslated region of $P P P 2 R 2 B$, which maps on chromosome $5 \mathrm{q} 32$ and encodes a brain-specific regulatory subunit of the phosphatase PP2A.[60,61] Individuals with more than 46 repeats on one allele develop the disease.[62] Rather than the gait ataxia typical of SCAs, SCA- $P P P 2 R 2 B$ often presents with prominent or isolated upperlimb and sometimes head action tremor (Video 1, Segment 1).[60] SCA-PPP2R2B is particularly common among the Agarwal community from northern India, but also reported in European, American, and nonIndian Asian kindreds.[63] Onset is generally in the fourth decade. Disease progression is slow and patients display varied clinical manifestations, including cerebellar signs (i.e., gait ataxia, limb dysmetria, dysarthria, abnormal eye movements), parkinsonism, dystonia, hyperreflexia, psychiatric symptoms, and, in later stages, cognitive decline.[63] Cerebral and cerebellar atrophy are typical MRI findings.[63] Testing for SCA$P P P 2 R 2 B$ should be considered in individuals with ET-like tremor and a positive family history of cerebellar, parkinsonian, pyramidal, and cognitive manifestations, especially if geographical provenance and brain imaging are consistent. Despite being an ET phenocopy at onset, SCA-PPP2R2B has not been associated with ET in a large cohort study.[64]

A heterozygous mutation in the gene encoding fibroblast growth factor 14 (FGF14) on chromosome 13q34 cause SCA-FGF14 (SCA27), which usually manifests with childhood-onset hand "trembling" (highfrequency, small-amplitude tremor) exacerbated by emotions and fatigue, and/or head titubation.[65] Tremor is the initial clinical feature in $60 \%$ of cases (and present in over 95\%), and may precede the appearance of progressive or episodic ataxia, dysarthria, nystagmus, orofacial dyskinesia, psychiatric manifestations and cognitive impairment by many years.[65] Mild to moderate cerebellar atrophy on MRI has been reported in $20 \%$ of cases, mainly older individuals.[59,65] The diagnosis should therefore be considered in patients with ataxia who report tremor since childhood, or other associated features, such as episodic ataxia and neuropsychiatric symptoms. 


\subsection{Autosomal dominant monogenic Parkinson's disease}

All genes causing monogenic Parkinson's disease (PD) may be linked with combined tremor syndromes, as rest tremor is a cardinal motor feature. Hand rest tremor in monogenic PD is indistinguishable from the classic pill-rolling tremor observed in idiopathic PD; unilateral leg rest tremor has been reported as a presenting feature in many PARK-LRRK2 (PARK8; chromosome 12q12), and may precede the appearance of other parkinsonian features by many years.[36,66-67] An overviews of monogenic PD is available elsewhere.[68]

\subsection{Neuroferritinopathy}

Neuroferritinopathy is an AD neurodegeneration with brain iron accumulation (NBIA) syndrome. Caused by mutations in the ferritin light chain gene (FTL; chromosome 19q13.33)[69], it was originally described in families from Northern England, but later also in France, Italy, Japan, and India. Neuroferritinopathy usually begins between the fourth and sixth decade of life with either prominent chorea, dystonia, pyramidal signs, or a bradykinetic-rigid syndrome. Onset with upper-limb postural and kinetic tremor, followed by gait difficulties and progressive cognitive impairment has been reported by several authors.[70-71] Palatal tremor was reported with cognitive decline in affected individuals of British and French ancestry.[70-73] Laboratory investigations reveal low to low-normal serum ferritin levels, with normal iron, hemoglobin and transferrin. Neuroradiological clues include cortical iron deposition (pencil lining sign),[74] bilateral pallidal necrosis, and cystic cavitation of the basal ganglia in advanced stages.[75]

\subsection{Tremor related to genes causing frontotemporal dementia with parkinsonism}

The genetic landscape of the syndrome of frontotemporal dementia with parkinsonism (and sometimes motor neuron disease) has been clarified in recent decades with the identification of multiple causative genes including MAPT, PRGN, TARDP, VCP, CHMP2B, and C9orf72.[76-82] In this context, parkinsonism generally manifests as an akinetic-rigid syndrome, with typical resting tremor being reported in less than $10 \%$ of cases.[77] FUS mutations rarely cause parkinsonism associated with a postural tremor resembling ET.[81]

\subsection{Hereditary geniospasm}

Hereditary geniospasm (HG) is an isolated tremor syndrome characterized by recurrent, paroxysmal elevation-and-depression of the chin and lower lip due to rhythmic mentalis muscle contractions at frequencies between $4-30 \mathrm{~Hz}$.[83,84] It is inherited as an $\mathrm{AD}$ trait with approximately $80 \%$ penetrance.[83] Episodes occur spontaneously or triggered by stress and last seconds to several hours. HG usually manifests soon after birth or in early childhood but frequency and severity of episodes generally decrease with age. Although the genes responsible for HG remain unknown, a genome-wide linkage analysis mapped some cases to chromosome 9q13-q21, and confirmed the genotypic heterogeneity of the disorder.[83] Despite its 
denomination, neurophysiological findings suggest that HG represents a focal brainstem myoclonus rather than tremor.[84]

\subsection{Autosomal dominant progressive ataxia with palatal tremor}

PAPT is a rare clinical syndrome characterized by progressive cerebellar dysfunction and low-frequency (1$2 \mathrm{~Hz}$ ) palatal tremor. While increasing evidence suggests that sporadic PAPT represents a novel 4-repeat tauopathy,[85] several genetic disorders are recognized to underlie familial cases of PAPT. These include late-onset Alexander disease,[86] SCA20[56] and SCA-ATXN7 (SCA7)[36,57] (see Table 2), the AR disorders GM2-gangliosidosis[87] and cerebrotendineous xantomatosis[88] (see Table 3), and mutations in the mitochondrial polymerase gamma gene ( $P O L G$; see section 5).[89]

Alexander disease is a rare AD leukodystrophy with age of onset from infancy to the early seventh decade. Adult-onset forms manifest with bulbar signs (dysarthria and dysphagia), spasticity, slowly progressive gait ataxia, dysautonomia, sleep disturbances, and cognitive impairment.[86,90] A 2-Hz palatal tremor is reported in around $40 \%$ of cases (Supplementary Table).[91] The disease is caused by mutations in GFAP, which maps on chromosome 17q21.31 and codes for the glial fibrillary acidic protein.[86] On brain MRI, almost 90\% of late-onset cases show severe medullary and cervical spinal cord atrophy, with a preserved basis pontis ('tadpole sign').[92] Although white matter abnormalities may coexist, lesion load is never as high as in early-onset disease.[86]

\subsection{Autosomal dominant hereditary spastic paraplegias}

Tremor rarely forms part of the spectrum of hereditary spastic paraplegias (HSPs).[93] Orthostatic tremor has been reported as a presenting or additional sign in three independent cases of HSP-REEP1 (HSP31; Supplementary Table).[36,94,95] Furthermore, hand tremor has been described in HSP-SPAST (HSP4). [36,93]

Table 2 provides summaries of the above-mentioned and other AD tremor disorders.[41-86,89-102]

\section{INHERITED AUTOSOMAL RECESSIVE TREMOR DISORDERS}

\subsection{Wilson's disease}

Wilson's disease (WD) is an AR disorder of copper metabolism caused by biallelic mutations in ATP7B (chromosome 13q14.3),[103] which results in defective copper excretion into the biliary tract, and subsequent toxic accumulation of free copper in different organs, primarily the liver and brain. Hepatic WD typically presents in late childhood, whereas neurological manifestations generally occur in the second or third decade (though cases presenting as young as 6 and as old as 72 years have been reported).[104] Tremor is the presenting manifestation in up to $55 \%$ of neurological WD, and may be accompanied by dystonia, 
parkinsonism, choreoathetosis, and cerebellar signs with dysphagia, dysarthria, and drooling.[103,104] Although well-known, the classic "wing-beating" tremor of the upper extremities is not the sole tremor type in WD (Supplementary Table). An ET-like tremor involving the arms, head and legs can be the earliest manifestation of neurological WD, as can tongue tremor with dysarthria.[103-106] WD-related tremors (which can be unilateral) may also be 'rubral-like', dystonic or parkinsonian and are generally accompanied by prominent postural and/or kinetic component. Investigations reveal low serum ceruloplasmin, excessive urinary copper excretion, cerebral atrophy with hyperintensity of the basal ganglia on T2-weighted MRI sequences, and Kayser-Fleischer rings. Liver biopsy may confirm the clinical suspicion in challenging cases.[103,104]

\subsection{Autosomal recessive Parkinson's disease and primary dystonias}

Overviews on monogenic PD and primary isolated dystonias are detailed elsewhere.[41-43,45,68] Among AR PD, PARK-Parkin (PARK2), PARK-PINK1 (PARK6), PARK-DJ-1 (PARK7), PARK-FBXO7 (PARK15), PARK-DNAJC6 (PARK19), and PARK-SYNJ1 (PARK20) may manifest with dystonic and/or parkinsonian rest tremor usually before the age of 40.[36,107-111] DYT/PARK-SLC6A3 causes infancy-, childhood-, and rarely adult-onset parkinsonism-dystonia with tremor.[36,112] An entity called benign tremulous parkinsonism of the young, characterized by rest and/or postural tremor in the legs or hands, sometimes as the sole or prominent feature of PD, has been reported in juvenile PARK-Parkin.[113] PLA2G6- and ATP13A2-related disorders can present as an AR parkinsonian syndrome, including rest tremor, and are reported in details below.[114] AR isolated dystonias related to the HPCA, COL6A3, and VPS16 genes are extremely rare. Mutations in the THAP1 (DYT6) or GNAL (DYT25) genes, which are usually dominantly inherited, can also be inherited in an AR manner.[45]

\subsection{Autosomal recessive syndromes of neurodegeneration with brain iron accumulation}

All genes linked to AR NBIA disorders may underlie combined tremor syndromes, including PANK2, PLA2G6, C19orf12, COASY, FA2H, ATP13A2, and CP.[114] Isolated rest or dystonic tremor of the upper limbs is reported as the presenting sign in atypical cases of NBIA/DYT-PANK2 (PKAN) with late onset (second to fourth decade).[36,115-119] In atypical cases of NBIA/DYT/PARK-PLA2G6 (PLAN) with onset in late adolescence or early twenties with dystonia-parkinsonism, pill-rolling tremor is present in approximately two thirds of cases along with psychiatric manifestations and rapid cognitive decline.[114,120] The clinical picture of aceruloplasminemia, a disorder resulting from biallelic mutations in the gene coding for ceruloplasmin $(C P)$, encompasses different movement disorders including tremor, chorea, ataxia, and/or dystonia alongside psychiatric and cognitive changes, diabetes mellitus and retinopathy.[121,122] Laboratory investigations usually reveal absent or very low serum ceruloplasmin with normal copper excretion and high ferritin. On MRI, iron deposition may be detected in the basal ganglia, red nucleus, and dentate, but also in the liver.[121,122] Intention tremor or parkinsonism with rest tremor have been described alongside dystonia, spasticity, cerebellar signs, neuropathy, and visual failure in 
mitochondrial membrane protein-associated neurodegeneration (MPAN), a childhood-onset NBIA syndrome caused by biallelic mutations in C19orf12 (chromosome 19q12).[123,124] Recently, AD mode of inheritance has also been reported for MPAN.[125]

\subsection{Autosomal recessive inborn errors of metabolism}

A plethora of AR neurometabolic diseases include tremor in their clinical phenotype,[126] including glutaric aciduria type I, classic galactosemia, and phenylketonuria. In these conditions, tremor may appear in early childhood or as late-onset sequela, regardless of prompt initiation of dietary treatment (Supplementary Table).[126,127] Glutaric aciduria type I is a disorder of lysine and tryptophan metabolism due to mutations in the glutaryl-CoA dehydrogenase gene ( $G C D H$; chromosome 19p13). Although the classic phenotype encompasses acute encephalopathic crises precipitated by febrile episodes before the age of 2 , bibrachial dystonic tremor with orofacial dyskinesias starting from adolescence or early adulthood have been reported in otherwise asymptomatic individuals.[128] Classic galactosemia is an inborn error of galactose metabolism caused by pathogenic variants in the galactose-1-phospate uridylyltransferase gene (GALT; chromosome 9p13). Acute systemic complications are typical of disease presentation in newborns. However, neurological manifestations, including action tremor of the arms and head, dystonia, cerebellar and pyramidal signs, as well as late-onset complications (e.g., premature ovarian failure (POF)) have been reported despite strict dietary galactose restriction since birth.[129]

Table 3 provides brief summaries of the above-mentioned and other AR tremor disorders.[87,88,93,103-136]

\section{INHERITED X-LINKED TREMOR DISORDERS}

\subsection{Fragile $X$-associated tremor ataxia syndrome}

FXTAS is a late-onset neurodegenerative disorder affecting around $40 \%$ of males carrying a premutation (CGG trinucleotide expansion of 55-200 repeats) allele in the 5' untranslated region of fragile X mental retardation gene (FMR1; chromosome Xq27.3). Estimated prevalence is 1:800 in males and 1:250 in females.[137,138] Onset is generally in the seventh decade with progressive disabling tremor and ataxia, variably associated with parkinsonism, cognitive and behavioral impairment, autonomic dysfunction, peripheral neuropathy, and sleep disturbances. Tremor is the earliest sign in nearly $80 \%$ of cases and may precede other symptoms by years. Documented tremor phenotypes include ET-like tremor affecting the hands, axial tremor, and non-isolated unilateral upper-limb rest tremor (Video 1, Segment 2).[138] Due to stochastic X-chromosome inactivation, female premutation carriers rarely develop FXTAS, though a case series reports tremor in nearly $40 \%$ of females permutation carriers, and many suffer from POF and depression.[139] A FXTAS-like phenotype has also been reported in fragile X syndrome (>200 repeats). In FXTAS, brain MRI shows T2 hyperintensities in the periventricular white matter and middle cerebellar 
peduncles (MCP sign) in 60\% of male and 13\% of female carriers.[138] The MCP sign on brain MRI along with a family history of mental retardation in male offspring and/or of amenorrhea before the age of 40 in females should prompt fragile $\mathrm{X}$ carrier testing.

\subsection{Lubag disease}

Lubag disease (DYT/PARK-TAF1) is an X-linked dystonia-parkinsonism described in males of maternal Filipino descent. Mutations in the noncoding regions within, and close to, the TATA-binding proteinassociated factor-1 gene (TAF1; chromosome Xq13.1) have been reported as cause of the disease.[140] Age of onset ranges from 12 to 79 years.[141] The classic phenotype is one of focal cranio-cervical dystonia that spreads to other body regions over time, and may combine with, or be replaced by, parkinsonism. Tremor and parkinsonism are common at presentation and may precede or overshadow dystonic features.[142] Tremor in DYT/PARK-TAF1 dystonia affects the head or limbs, and may appear at rest, on posture and/or during movements. It is often asymmetric and can be misdiagnosed as PD or ET. Lubag patients may also exhibit myorhythmia.[141] Female carriers are mostly asymptomatic, though a small minority manifest dystonia, parkinsonism, and/or chorea. Lubag disease should be suspected when an isolated or combined tremor syndrome occurs in individuals of Filipino ancestry and there is a family history of parkinsonism and/or dystonia consistent with X-linked inheritance.[141]

Table 4 provides brief summaries of the above-mentioned and other X-linked tremor disorders.[137-147]

\section{INHERITED MITOCHONDRIAL TREMOR DISORDERS}

Defects in maternally inherited mitochondrial DNA (mtDNA) or in nuclear genes (nDNA) encoding respiratory chain subunits may result in isolated and combined tremor syndromes.[89,148-152] Mutations in $P O L G$ (nDNA) may cause early-onset levodopa-responsive parkinsonism with typical rest tremor with either dominant or recessive inheritance. $P O L G$-related parkinsonism usually begins around age 40 , but onset has been reported as early as the third decade in some families. POLG mutations may also underlie familial PAPT.[89,148] A task-specific dystonic tremor was the presenting feature in a patient with pathogenic variant in SURF1-related Leigh syndrome (nDNA).[149] A C10orf2 TWINKLE variant has been reported in a patient presenting with simultaneous orthostatic tremor and eyelid ptosis.[150] Cytochrome c oxidase deficiency due to mutation in COX20 gene (mtDNA) causes a childhood-onset cerebellar ataxia with intention tremor and pyramidal signs.[151] An extensive review of mitochondrial disorders is covered elsewhere.[149,151,152] 


\section{TREMOR DISORDERS RELATED TO CHROMOSOMAL ABNORMALITIES}

\section{Sex chromosome aneuploidies}

Tremor is a common manifestation of sex chromosome aneuploidies, including KS, Jacobs syndrome (JS), and rarer supernumerary X or Y syndromes (Supplementary Table).[153-159]

KS is the most frequent sex chromosome abnormality among males (1:700 newborns) and the most common cause of infertility in the male population. The clinical picture includes tall stature from puberty, arm span exceeding height, and hypergonadotropic hypogonadism/androgen deficiency.[153] Intention tremor of the upper extremities, sometimes associated with head, voice, and leg tremor, has been reported in up to $75 \%$ of individuals with KS (47,XXY karyotype).[154,155]

Unilateral or bilateral rest, postural and/or intention tremor of the arms as well as head tremor is also recognized in JS (47,XYY karyotype). Despite a prevalence of 1:1,000 in the male population, it is estimated that up to $88 \%$ of cases remain undiagnosed in their lifetime.[153,156] Clinical clues include tall stature, central adiposity, macrocephaly, macro-orchidism, hypotonia, clinodactyly, and hypertelorism. Half of males with XYY karyotype receive a diagnosis of attention deficit or autistic spectrum disorder.[156]

Postural and/or action tremor of the upper limbs with onset during the first or second decade is reported in 48,XXYY syndrome, which has an estimated prevalence of approximately 1:18,000-1:40,000 among newborn males. Physical features are similar to those seen in KS, although 48,XXYY patients exhibit significantly more neurodevelopmental and psychological features, including dysmorphic features (e.g., long face, epicanthal folds, poor dentition), pes planus, attention deficit disorder, and learning difficulties.[153,157-159]

In summary, males presenting with tremor from an early age with suggestive physical features (tall stature, micro- or macro-orchidism, and rarely, facial dysmorphism), and a history of infertility and/or early developmental delays or learning disabilities should prompt karyotyping to evaluate for sex chromosome aneuploidies.[153-159] As aneuploidies are not inherited, family history is usually unremarkable.

Table 5 provides brief summaries of tremor disorders related to sex chromosome aneuploidies and structural chromosomal abnormalities.[153-161]

\section{DISCUSSION}

A plethora of gene and chromosomal abnormalities are known to cause isolated and combined tremor syndromes. Details on genetic disorders causing tremor and relevant clues to diagnosis are reported in Tables 2 to 5 . Figure 1 provides a phenotype-to-genotype algorithm to guide clinicians in the differential diagnosis and genetic testing options. 
Most genetic tremor disorders exhibit a complex clinical picture when their phenotype is fully expressed. However, some (e.g., DYT-ANO3, SCA-PPP2R2B, FXTAS, KS) may resemble ET both at onset and for many years thereafter. Moreover, some tremor syndromes of uninvestigated or undetermined etiology may be erroneously referred to as ET in clinical practice. By providing a detailed syndromic approach to tremors and strict diagnostic criteria for ET, the 2018 tremor classification allows stratification of patients into phenotypically homogeneous subgroups, and may ultimately contribute to further advances in our understanding of the genetic basis of tremors.

Deconstructing Axis 1 of the recent classification,[1] the differential diagnosis of presumed genetic tremors appears to be largely based on the presence or absence of associated signs, as illustrated in Figure 1. Young age of onset narrows the differential diagnosis, as few genetic tremor disorders manifest in childhood or adolescence (e.g., WD, KS, some monogenic primary dystonias and neurometabolic diseases). Specific patterns of body distribution can aid the diagnostic process (e.g., palatal tremor: Alexander disease, SCA20, $P O L G$-related disorders; laryngeal tremor: dystonic conditions, ET). Similarly, rest tremor limits the differential diagnoses mostly to parkinsonian conditions, while intention tremor should prompt a search for other signs of cerebellar involvement, as seen in SCAs and other ataxic syndromes. Abnormal laboratory tests may hint at the diagnosis. These include serum ceruloplasmin and urinary copper levels in WD and aceruloplasminemia, iron studies in neuroferritinopathy and aceruloplasminemia, sex hormone levels in sex chromosome aneuploidies, and CSF analysis in dopa-responsive dystonia and rarer neurometabolic diseases. Specific imaging findings on brain MRI in FXTAS, WD, NBIA, and positive dopamine receptor imaging in PD and other parkinsonian condition can also be directive.

It is noteworthy that genetic tremor syndromes often occur in the absence of a positive family history of tremor. Although being particularly frequent for AR, X-linked, and classic mitochondrial inheritance, this may also occur in $\mathrm{AD}$ disorders with incomplete or delayed penetrance, or due to de novo mutations. Moreover, sex chromosome aneuploidies are not inherited, and parents of a child with one of these conditions are not at an increased risk of having a second affected child. Geographical provenance may prove useful, suggesting diagnoses such as SCA-PPP2R2B, DYT/PARK-TAF1, and neuroferritinopathy. A history of intellectual disability in male offspring and/or premature amenorrhea in female family members should prompt clinical suspicion of FXTAS.

Despite the large number of genetic defects which are recognized to cause tremor disorders, a substantial proportion of patients with tremor-dominant clinical pictures encountered in the routine care remain without a definitive molecular diagnosis, even after undergoing NGS-based investigations, such as multigene panels, whole exome sequencing (WES) and whole genome sequencing (WGS).

An exponential growth of genetic knowledge on tremors is expected with the progress and widespread availability of NGS and LRS technologies.[38,39,162-164] Firstly, targeted gene panels allow simultaneous cost-effective testing for several known tremor-related genes and simplify molecular diagnosis of sporadic cases due to de novo mutations, when a priori hypotheses on responsible genes may be challenging. Secondly, WES or WGS combined with Sanger sequencing and linkage analysis may identify new genes 
responsible for tremor syndromes in individual families. Thirdly, NGS makes large scale exome or genome screening possible, in order to identify new risk loci through approaches such as genome-wide association studies. Finally, LRS may enable the detection of variants in NGS blind genomic spots, reveal large and complex rearrangements, large insertions or deletions, haplotype phasing, and changes in DNA methylation.[38,39] NGS and LRS technologies may therefore shed light on heterogeneous etiologies underlying tremor syndromes currently labeled as ET, and expand the genotypic spectrum of rarer or more complex tremor disorders.[38,39,163,164] Continuous updating on genotype and phenotype of tremor syndromes will be possible with online resources, such as Online Mendelian Inheritance in Man® (https://www.omim.org/), Human GeneReviews® (https://www.ncbi.nlm.nih.gov/books/NBK1116/), Genecards ${ }^{\circledR}$ (http://www.genecards.org), and Movement Disorder Society Genetic mutation database (https://www.mdsgene.org). Nevertheless, thorough phenotyping of patients and targeted investigations remain the cornerstone of clinical decision-making for genetic testing and result interpretation.

All patients provided written informed consent to be videotaped, and to the publication of the videos in both the printed and online modalities. 


\section{Acknowledgements}

Francesca Magrinelli is supported by the European Academy of Neurology (EAN) Research Fellowship 2020.

\section{Funding}

This research did not receive any specific grant from funding agencies in the public, commercial, or not-forprofit sectors 


\section{References}

[1] K.P. Bhatia, P. Bain, N. Bajaj, R.J. Elble, M. Hallett, E.D. Louis, J. Raethjen, M. Stamelou, C.M. Testa, G. Deuschl; Tremor Task Force of the International Parkinson and Movement Disorder Society, Consensus statement on the classification of tremors. From the task force on tremor of the International Parkinson and Movement Disorder Society, Mov. Disord. 33 (2018) 75-87.

[2] G. Deuschl, P. Bain, M. Brin, Consensus statement of the Movement Disorder Society on tremor. Ad hoc scientific committee, Mov. Disord 13 Suppl. 3 (1998): 2-23.

[3] E.D. Louis, J.J. Ferreira, How common is the most common adult movement disorder? Update on the worldwide prevalence of essential tremor, Mov. Disord. 25 (2010): 534e541.

[4] E.D. Louis, O. Dogu, Does age of onset in essential tremor have a bimodal distribution? Data from a tertiary referral setting and a population-based study, Neuroepidemiology 29 (2007): 208-212.

[5] F. Hopfner, D. Haubenberger, W.R. Galpern, K. Gwinn, A. Van't Veer, S. White, K. Bhatia, C.H. Adler, D. Eidelberg, W. Ondo, G.T. Stebbins, C.M. Tanner, R.C. Helmich, F.A. Lenz, R.V. Sillitoe, D. Vaillancourt, J.L. Vitek, E.D. Louis, H.A. Shill, M.P. Frosch, T. Foroud, G. Kuhlenbäumer, A. Singleton, C.M. Testa, M. Hallett, R. Elble, G. Deuschl G, Knowledge gaps and research recommendations for essential tremor, Parkinsonism Relat. Disord. 33 (2016): 27-35.

[6] J.R. Gulcher, P. Jónsson, A. Kong, K. Kristjánsson, M.L. Frigge, A. Kárason, I.E. Einarsdóttir, H. Stefánsson, A.S. Einarsdóttir, S. Sigurthoardóttir, S. Baldursson, S. Björnsdóttir, S.M. Hrafnkelsdóttir, F. Jakobsson, J. Benedickz, K. Stefánsson, Mapping of a familial essential tremor gene, FET1, to chromosome 3q13, Nat. Genet. 17 (1997): 84-87.

[7] J.J. Higgins, L.T. Pho, L.E. Nee, A gene (ETM) for essential tremor maps to chromosome 2p22-p25, Mov. Disord. 12 (1997): 859-864.

[8] A. Sazci, E. Ergul, K. Bayulkem, Association of the C677T and A1298C polymorphisms of methylenetetrahydrofolate reductase gene in patients with essential tremor in Turkey, Mov. Disord. 19 (2004): 1472-1476.

[9] J.J. Higgins, R.Q. Lombardi, J. Pucilowska, J. Jankovic, E.K. Tan, J.P. Rooney, A variant in the HS1BP3 gene is associated with familial essential tremor, Neurology 64 (2005): 417-421. 
[10] A. Shatunov, N. Sambuughin, J. Jankovic, R. Elble, H.S. Lee, A.B. Singleton, A. Dagvadorj, J. Ji, Y. Zhang, V.E. Kimonis, J. Hardy, M. Hallett, L.G. Goldfarb, Genomewide scans in North American families reveal genetic linkage of essential tremor to a region on chromosome 6p23, Brain 129 (2006): 2318-2331.

[11] G. Lucotte, J.P. Lagarde, B. Funalot, P. Sokoloff, Linkage with the Ser9Gly DRD3 polymorphism in essential tremor families, Clin. Genet. 69 (2006): 437-440.

[12] H. Stefansson, S. Steinberg, H. Petursson, O. Gustafsson, I.H. Gudjonsdottir, G.A. Jonsdottir, S.T. Palsson, T. Jonsson, J. Saemundsdottir, G. Bjornsdottir, Y. Böttcher, T. Thorlacius, D. Haubenberger, A. Zimprich, E. Auff, C. Hotzy, C.M. Testa, L.A. Miyatake, A.R. Rosen, K. Kristleifsson, D. Rye, F. Asmus, L. Schöls, M. Dichgans, F. Jakobsson, J. Benedikz, U. Thorsteinsdottir, J. Gulcher, A. Kong, K. Stefansson, Variant in the sequence of the LINGO1 gene confers risk of essential tremor, Nat. Genet. 41 (2009): 277-279. Erratum in: Nat. Genet. 41 (2009): 504.

[13] C. Vilariño-Güell, A.I. Soto-Ortolaza, A. Rajput, D.C. Mash, S. Papapetropoulos, R. Pahwa, K.E. Lyons, R.J. Uitti, Z.K. Wszolek, D.W. Dickson, M.J. Farrer, O.A. Ross, MAPT H1 haplotype is a risk factor for essential tremor and multiple system atrophy, Neurology 76 (2011): 670-672.

[14] S. Their, D. Lorenz, M. Nothnagel, C. Poremba, F. Papengut, S. Appenzeller, S. Paschen, F. Hofschulte, A.C. Hussl, S. Hering, W. Poewe, F. Asmus, T. Gasser, L. Schöls, K. Christensen, A. Nebel, S. Schreiber, S. Klebe, G. Deuschl, G. Kuhlenbäumer, Polymorphisms in the glial glutamate transporter SLC1A2 are associated with essential tremor, Neurology 79 (2012): 243-248.

[15] N.D. Merner, S.L. Girard, H. Catoire, C.V. Bourassa, V.V. Belzil, J.B. Rivière, P. Hince, A. Levert, A. Dionne-Laporte, D. Spiegelman, A. Noreau, S. Diab, A. Szuto, H. Fournier, J. Raelson, M. Belouchi, M. Panisset, P. Cossette, N. Dupré, G. Bernard, S. Chouinard, P.A. Dion, G.A. Rouleau, Exome sequencing identifies FUS mutations as a cause of essential tremor, Am. J. Hum. Genet. 91 (2012): 313-319.

[16] H. Unal Gulsuner, S. Gulsuner, F.N. Mercan, O.E. Onat, T. Walsh, H. Shahin, M.K. Lee, O. Dogu, T. Kansu, H. Topaloglu, B. Elibol, C. Akbostanci, M.C. King, T. Ozcelik, A.B. Tekinay, Mitochondrial serine protease HTRA2 p.G399S in a kindred with essential tremor and Parkinson disease, Proc. Natl. Acad. Sci. USA 111 (2014): 18285-18290. 
[17] H. Hor, L. Francescatto, L. Bartesaghi, S. Ortega-Cubero, M. Kousi, O. Lorenzo-Betancor, F.J. Jiménez-Jiménez, A. Gironell, J. Clarimón, O. Drechsel, J.A. Agúndez, D. Kenzelmann Broz, R. Chiquet-Ehrismann, A. Lleó, F. Coria, E. García-Martin, H. Alonso-Navarro, M.J. Martí, J. Kulisevsky, C.N. Hor, S. Ossowski, R. Chrast, N. Katsanis, P. Pastor, X. Estivill, Missense mutations in TENM4, a regulator of axon guidance and central myelination, cause essential tremor, Hum. Mol. Genet. 24 (2015): 5677-5686.

[18] S. Ortega-Cubero, O. Lorenzo-Betancor, E. Lorenzo, J.A. Agúndez, F.J. Jiménez-Jiménez, O.A. Ross, I. Wurster, C. Mielke, J.J. Lin, F. Coria, J. Clarimon, M. Ezquerra, L. Brighina, G. Annesi, H. AlonsoNavarro, E. García-Martin, A. Gironell, M.J. Marti, K.C. Yueh, Z.K. Wszolek, M. Sharma, D. Berg, R. Krüger, M.A. Pastor, P. Pastor, TREM2 R47H variant and risk of essential tremor: a cross-sectional international multicenter study, Parkinsonism Relat. Disord. 21 (2015): 306-309.

[19] E. Sánchez, A. Bergareche, C.E. Krebs, A. Gorostidi, V. Makarov, J. Ruiz-Martinez, A. Chorny, A. Lopez de Munain, J.F. Marti-Masso, C. Paisán-Ruiz, SORT1 Mutation resulting in sortilin deficiency and p75(NTR) upregulation in a family with essential tremor, ASN Neuro. 7 (2015).

[20] A. Bergareche, M. Bednarz, E. Sánchez, C.E. Krebs, J. Ruiz-Martinez, P. De La Riva, V. Makarov, A. Gorostidi, K. Jurkat-Rott, J.F. Marti-Masso, C. Paisán-Ruiz, SCN4A pore mutation pathogenetically contributes to autosomal dominant essential tremor and may increase susceptibility to epilepsy, Hum. Mol. Genet. 24 (2015): 7111-7120.

[21] A. Rajput, J.P. Ross, C.Q. Bernales, S. Rayaprolu, A.I. Soto-Ortolaza, O.A. Ross, J. van Gerpen, R.J. Uitti, Z.K. Wszolek, A.H. Rajput, C. Vilariño-Güell, VPS35 and DNAJC13 disease-causing variants in essential tremor, Eur. J. Hum. Genet. 23 (2015): 887-888.

[22] X. Liu, N. Hernandez, S. Kisselev, A. Floratos, A. Sawle, I. Ionita-Laza, R. Ottman, E.D. Louis, L.N. Clark, Identification of candidate genes for familial early-onset essential tremor, Eur. J. Hum. Genet. 24 (2016): 1009-1015.

[23] S.H. Müller, S.L. Girard, F. Hopfner, N.D. Merner, C.V. Bourassa, D. Lorenz, L.N. Clark, L. Tittmann, A.I. Soto-Ortolaza, S. Klebe, M. Hallett, S.A. Schneider, C.A. Hodgkinson, W. Lieb, Z.K. Wszolek, M. Pendziwiat, O. Lorenzo-Betancor, W. Poewe, S. Ortega-Cubero, K. Seppi, A. Rajput, A. Hussl, A.H. Rajput, D. Berg, P.A. Dion, I. Wurster, J.M. Shulman, K. Srulijes, D. Haubenberger, P. 
Pastor, C. Vilariño-Güell, R.B. Postuma, G. Bernard, K.H. Ladwig, N. Dupré, J. Jankovic, K. Strauch, M. Panisset, J. Winkelmann, C.M. Testa, E. Reischl, K.E. Zeuner, O.A. Ross, T. Arzberger, S.

Chouinard, G. Deuschl, E.D. Louis, G. Kuhlenbäumer, G.A. Rouleau, Genome-wide association study in essential tremor identifies three new loci, Brain 139 (2016): 3163-3169.

[24] X.R. Leng, X.H. Qi, Y.T. Zhou, Y.P. Wang, Gain-of-function mutation p.Arg225Cys in SCN11A causes familial episodic pain and contributes to essential tremor, J. Hum. Genet. 62 (2017): 641-646.

[25] L. Yuan, X. Deng, Z. Song, S. Deng, W. Zheng, P. Mao, H. Deng, Systematic analysis of genetic variants in patients with essential tremor, Brain Behav. 8 (2018): e01100.

[26] A. Sazci, N. Uren, H.A. Idrisoglu, E. Ergul, The rs2228570 variant of the vitamin D receptor gene is associated with essential tremor, Neurosci. Bull. 35 (2019): 362-364.

[27] J. Chen, P. Huang, Y. He, J. Shen, J. Du, S. Cui, S. Chen, J. Ma, IL1B polymorphism is associated with essential tremor in Chinese population, BMC Neurol. 19 (2019): 99.

[28] Z. Odgerel, S. Sonti, N. Hernandez, J. Park, R. Ottman, E.D. Louis, L.N. Clark, Whole genome sequencing and rare variant analysis in essential tremor families, PLoS One 14 (2019): e0220512.

[29] Q.Y. Sun, Q. Xu, Y. Tian, Z.M. Hu, L.X. Qin, J.X. Yang, W. Huang, J. Xue, J.C. Li, S. Zeng, Y. Wang, H.X. Min, X.Y. Chen, J.P. Wang, B. Xie, F. Liang, H.N. Zhang, C.Y Wang, L.F. Lei, X.X. Yan, H.W. Xu, R.H. Duan, K. Xia, J.Y. Liu, H. Jiang, L. Shen, J.F. Guo, B.S. Tang, Expansion of GGC repeat in the human-specific NOTCH2NLC gene is associated with essential tremor, Brain 143 (2020): 222-233.

[30] L.J. Findley, W.C. Koller, Definitions and behavioural classifications, in: L.J. Findley, W.C. Koller (Eds), Handbook of Tremor Disorders, Marcel Dekker, New York, 1995, pp. 1-5.

[31] E.D. Louis, B. Ford, H. Lee, H. Andrews, G. Cameron, Diagnostic criteria for essential tremor. A population perspective, Arch. Neurol. 55 (1998): 823-828.

[32] R.J. Elble, Diagnostic criteria for essential tremor and differential diagnosis, Neurology 54 Suppl. 4 (2000): S2-S6.

[33] M.F. Brin, W. Koller, Epidemiology, genetics of essential tremor, Mov. Disord. 13 Suppl. 3 (1998): S55-S63. 
[34] E.D. Louis, R. Ottman, B. Ford, S. Pullman, M. Martinez, S. Fahn, W.A. Hauser, The Washington Heights-Inwood Genetic Study of Essential Tremor: methodologic issues in essential-tremor research, Neuroepidemiology 16 (1997): 124-133.

[35] F. Hopfner, R.C. Helmich, The etiology of essential tremor: genes versus environment, Parkinsonism Relat. Disord. 46 (2018): S92-S96.

[36] C. Marras, A. Lang, B.P. van de Warrenburg, C.M. Sue, S.J. Tabrizi, L. Bertram, S. MercimekMahmutoglu, D. Ebrahimi-Fakhari, T.T. Warner, A. Durr, B. Assmann, K. Lohmann, V. Kostic, C. Klein, Nomenclature of genetic movement disorders: Recommendations of the International Parkinson and Movement Disorder Society task force, Mov. Disord. 32 (2017): 724-725.

[37] V. Tam, N. Patel, M. Turcotte, Y. Bossé, G. Paré, D. Meyre, Benefits and limitations of genome-wide association studies, Nat. Rev. Genet. 20 (2019): 467-484.

[38] T. Mantere, S. Kersten, A. Hoischen, Long-Read Sequencing Emerging in Medical Genetics, Front. Genet. 10 (2019): 426.

[39] M.K. Midha, M. Wu, K.P. Chiu, Long-read sequencing in deciphering human genetics to a greater depth, Hum. Genet. 138 (2019): 1201-1215.

[40] A. Albanese, K. Bhatia, S.B. Bressman, M.R. Delong, S. Fahn, V.S. Fung, M. Hallett, J. Jankovic, H.A. Jinnah, C. Klein, A.E. Lang, J.W. Mink, J.K. Teller, Phenomenology and classification of dystonia: a consensus update, Mov. Disord. 28 (2013): 863-873.

[41] Müller U, The monogenic primary dystonia, Brain 132 (2009): 2005-2025.

[42] B. Balint, K.P. Bhatia, Isolated and combined dystonia syndromes - an update on new genes and their phenotypes, Eur. J. Neurol. 22 (2015): 610-617.

[43] M. Zech, D.D. Lam, L. Francescatto, B. Schormair, A.V. Salminen, A. Jochim, T. Wieland, P. Lichtner, A. Peters, C. Gieger, H. Lochmüller, T.M. Strom, B. Haslinger, N. Katsanis, J. Winkelmann, Recessive mutations in the alpha-3 (VI) collagen gene COL6A3 cause early-onset isolated dystonia, Am. J. Hum. Genet. 96 (2015): 883-893.

[44] N.E. Mencacci, I. Rubio-Agusti, A. Zdebik, F. Asmus, M.H. Ludtmann, M. Ryten, V. Plagnol, A.K. Hauser, S. Bandres-Ciga, C. Bettencourt, P. Forabosco, D. Hughes, M.M. Soutar, K. Peall, H.R. Morris, D. Trabzuni, M. Tekman, H.C. Stanescu, R. Kleta, M. Carecchio, G. Zorzi, N. Nardocci, B. 
Garavaglia, E. Lohmann, A. Weissbach, C. Klein, J. Hardy, A.M. Pittman, T. Foltynie, A.Y. Abramov, T. Gasser, K.P. Bhatia, N.W. Wood, A missense mutation in KCTD17 causes autosomal dominant myoclonus-dystonia, Am. J. Hum. Genet. 96 (2015): 938-947.

[45] B. Balint, N.E. Mencacci, E.M. Valente, A. Pisani, J. Rothwell, J. Jankovic, M. Vidailhet, K.P. Bhatia, Dystonia, Nat. Rev. Dis. Primers 4 (2018): 25.

[46] G. Charlesworth, V. Plagnol, K.M. Holmström, J. Bras, U.M. Sheerin, E. Preza, I. Rubio-Agusti, M. Ryten, S.A. Schneider, M. Stamelou, D. Trabzuni, A.Y. Abramov, K.P. Bhatia, N.W. Wood, Mutations in ANO3 cause dominant craniocervical dystonia: ion channel implicated in pathogenesis, Am. J. Hum. Genet. 91 (2012): 1041-1050.

[47] A. Münchau, E.M. Valente, M.B. Davis, V. Stinton, N.W. Wood, N.P. Quinn, K.P. Bhatia, A Yorkshire family with adult-onset cranio-cervical primary torsion dystonia, Mov. Disord. 15 (2000): 954-959.

[48] P.R. Blackburn, M.T. Zimmermann, J.M. Gass, K.G. Harris, M.A. Cousin, N.J. Boczek, O.A. Ross, E.W. Klee, P.W. Brazis, J.A. Van Gerpen, P.S. Atwal, A novel ANO3 variant identified in a 53-yearold woman presenting with hyperkinetic dysarthria, blepharospasm, hyperkinesias, and complex motor tics, BMC Med. Genet. 17 (2016): 93.

[49] M. Stamelou, G. Charlesworth, C. Cordivari, S.A. Schneider, G. Kägi, U.M. Sheerin, I. Rubio-Agusti, A. Batla, H. Houlden, N.W. Wood, K.P. Bhatia, The phenotypic spectrum of DYT24 due to ANO3 mutations, Mov. Disord. 29 (2014): 928-934.

[50] M. Segawa, A. Hosaka, F. Miyagawa, Y. Nomura, H. Imai, Hereditary progressive dystonia with marked diurnal fluctuation, Adv. Neurol. 14 (1976): 215-233.

[51] I. Trender-Gerhard, M.G. Sweeney, P. Schwingenschuh, P. Mir, M.J. Edwards, A. Gerhard, J.M. Polke, M.G. Hanna, M.B. Davis, N.W. Wood, K.P. Bhatia, Autosomal-dominant GTPCH1-deficient DRD: clinical characteristics and long-term outcome of 34 patients, J. Neurol. Neurosurg. Psychiatry 80 (2009): 839-845.

[52] S. Wijemanne, J. Jankovic, Dopa-responsive dystonia--clinical and genetic heterogeneity, Nat. Rev. Neurol. 11 (2015): 414-424. 
[53] E. O'Hearn, S.E. Holmes, P.C. Calvert, C.A. Ross, R.L. Margolis, SCA-12: tremor with cerebellar and cortical atrophy is associated with a CAG repeat expansion, Neurology 56 (2001): 299-303.

[54] H.J. Schelhaas, P.F. Ippel, G. Hageman, R.J. Sinke, E.N. van der Laan, F.A. Beemer, Clinical and genetic analysis of a four-generation family with a distinct autosomal dominant cerebellar ataxia, J. Neurol. 248 (2001): 113-120.

[55] J.C. van Swieten, E. Brusse, B.M. de Graaf, E. Krieger, R. van de Graaf, I. de Koning, A. MaatKievit, P. Leegwater, D. Dooijes, B.A. Oostra, P. Heutink, A mutation in the fibroblast growth factor 14 gene is associated with autosomal dominant cerebellar ataxia [corrected], Am. J. Hum. Genet. 72 (2003): 191-199.

[56] M.A. Knight, R.J. Gardner, M. Bahlo, T. Matsuura, J.A. Dixon, S.M. Forrest, E. Storey, Dominantly inherited ataxia and dysphonia with dentate calcification: spinocerebellar ataxia type 20, Brain 127 (2004): 1172-1181.

[57] G. Modi, M. Modi, I. Martinus, J. Rodda, D. Saffer, The clinical and genetic characteristics of spinocerebellar ataxia type 7 (SCA7) in three black South African families, Acta Neurol. Scand. 101 (2000): 177-182.

[58] M. Samuel, N. Torun, P.J. Tuite, J.A. Sharpe, A.E. Lang, Progressive ataxia and palatal tremor (PAPT): clinical and MRI assessment with review of palatal tremors, Brain 127 (2004): 1252-1268.

[59] J. van Gaalen, P. Giunti, B.P. van de Warrenburg, Movement disorders in spinocerebellar ataxias, Mov. Disord. 26 (2011): 792-800.

[60] S.E. Holmes, E. O'Hearn, R.L. Margolis, Why is SCA12 different from other SCAs?, Cytogenet. Genome Res. 100 (2003): 189-197.

[61] S.E. Holmes, E.E. O'Hearn, M.G. McInnis, D.A. Gorelick-Feldman, J.J. Kleiderlein, C. Callahan, N.G. Kwak, R.G. Ingersoll-Ashworth, M. Sherr, A.J. Sumner, A.H. Sharp, U. Ananth, W.K. Seltzer, M.A. Boss, A.M. Vieria-Saecker, J.T. Epplen, O. Riess, C.A. Ross, R.L. Margolis, Expansion of a novel CAG trinucleotide repeat in the 5 region of PPP2R2B is associated with SCA12, Nat. Genet. 23 (1999): 391-392.

[62] Y. Dong, J.J. Wu, Z.Y. Wu, Identification of 46 CAG repeats within PPP2R2B as probably the shortest pathogenic allele for SCA12, Parkinsonism Relat. Disord. 21 (2015): 398-401. 
[63] E. O'Hearn, S.E. Holmes, R.L. Margolis, Spinocerebellar ataxia type 12, Handb. Clin. Neurol. 103 (2012): 535-547.

[64] L.N. Clark, X. Ye, X. Liu, K. Mirzozoda, E.D. Louis, Genetic analysis of ten common degenerative hereditary ataxia loci in patients with essential tremor, Parkinsonism Relat. Disord. 21 (2015): 943947.

[65] C.L. Groth, B.D. Berman, Spinocerebellar ataxia 27: a review and characterization of an evolving phenotype, Tremor Other Hyperkinet. Mov. (NY) 8 (2018): 534.

[66] N.L. Khan, S. Jain, J.M. Lynch, N. Pavese, P. Abou-Sleiman, J.L. Holton, D.G. Healy, W.P. Gilks, M.G. Sweeney, M. Ganguly, V. Gibbons, S. Gandhi, J. Vaughan, L.H. Eunson, R. Katzenschlager, J. Gayton, G. Lennox, T. Revesz, D. Nicholl, K.P. Bhatia, N. Quinn, D. Brooks, A.J. Lees, M.B. Davis, P. Piccini, A.B. Singleton, N.W. Wood, Mutations in the gene LRRK2 encoding dardarin (PARK8) cause familial Parkinson's disease: clinical, pathological, olfactory and functional imaging and genetic data, Brain 128 (2005): 2786-2796.

[67] C. Paisàn-Ruìz, A. Sàenz, A. Lòpez de Munain, I. Martì, A. Martìnez Gil, J.F. Martì-Massò, J. PèrezTur, Familial Parkinson's disease: clinical and genetic analysis of four Basque families, Ann. Neurol. 57 (2005): 365-372.

[68] A. Puschmann, Monogenic Parkinson's disease and parkinsonism: clinical phenotypes and frequencies of known mutations, Parkinsonism Relat. Disord. 19 (2013): 407-415.

[69] A.R. Curtis, C. Fey, C.M. Morris, L.A. Bindoff, P.G. Ince, P.F. Chinnery, A. Coulthard, M.J. Jackson, A.P. Jackson, D.P. McHale, D. Hay, W.A. Barker, A.F. Markham, D. Bates, A. Curtis, J. Burn, Mutation in the gene encoding ferritin light polypeptide causes dominant adult-onset basal ganglia disease, Nat. Genet. 28 (2001), 350-354.

[70] P.F. Chinnery, D.E. Crompton, D. Birchall, M.J. Jackson, A. Coulthard, A. Lombès, N. Quinn, A. Wills, N. Fletcher, J.P. Mottershead, P. Cooper, M. Kellett, D. Bates, J. Burn, Clinical features and natural history of neuroferritinopathy caused by the FTL1 460InsA mutation, Brain 130 (2007): 110119. 
[71] E. Ohta, T. Nagasaka, K. Shindo, S. Toma, K. Nagasaka, K. Ohta, Z. Shiozawa, Neuroferritinopathy in a Japanese family with a duplication in the ferritin light chain gene, Neurology 70 (2008): 14931494.

[72] A. Kubota, A. Hida, Y. Ichikawa, Y. Momose, J. Goto, Y. Igeta, H. Hashida, K. Yoshida, S. Ikeda, I. Kanazawa, S. Tsuji, A novel ferritin light chain gene mutation in a Japanese family with neuroferritinopathy: description of clinical features and implications for genotype-phenotype correlations, Mov. Disord. 24 (2009): 441-445.

[73] A.J. Wills, G.V. Sawle, P.R. Guilbert, A.R. Curtis, Palatal tremor and cognitive decline in neuroferritinopathy, J. Neurol. Neurosurg. Psychiatry 73 (2002): 91-92.

[74] A. Batla, M.E. Adams, R. Erro, C. Ganos, B. Balint, N.E. Mencacci, K.P. Bhatia, Cortical pencil lining in neuroferritinopathy: a diagnostic clue, Neurology 84 (2015): 1816-1818.

[75] E. Ohta, Y. Takiyama, MRI findings in neuroferritinopathy, Neurol. Res. Int. (2012) 2012: 197438.

[76] J.F. Baizabal-Carvallo, J. Jankovic, Parkinsonism, movement disorders and genetics in frontotemporal dementia, Nat. Rev. Neurol. 12 (2016): 175-185.

[77] J. Diehl-Schmid, J. Schūlte-Overberg, J. Hartmann, H. Förstl, A. Kurz, P. Häussermann, Extrapyramidal signs, primitive reflexes and incontinence in fronto-temporal dementia, Eur. J. Neurol. 14 (2007): 860-864.

[78] J. Siuda, S Fujioka, ZK Wszolek, Parkinsonian syndrome in familial frontotemporal dementia, Parkinsonism Relat. Disord. 20 (2014): 957-964.

[79] S. Davion, N. Johnson, S. Weintraub, M.M. Mesulam, A. Engberg, M. Mishra, M. Baker, J. Adamson, M. Hutton, R. Rademakers, E.H. Bigio, Clinicopathologic correlation in PGRN mutations, Neurology 69 (2007): 1113-1121.

[80] B.F. Boeve, K.B. Boylan, N.R. Graff-Radford, M. DeJesus-Hernandez, D.S. Knopman, O. Pedraza, P. Vemuri, D. Jones, V. Lowe, M.E. Murray, D.W. Dickson, K.A. Josephs, B.K. Rush, M.M. Machulda, J.A. Fields, T.J. Ferman, M. Baker, N.J. Rutherford, J. Adamson, Z.K. Wszolek, A. Adeli, R. Savica, B. Boot, K.M. Kuntz, R. Gavrilova, A. Reeves, J. Whitwell, K. Kantarci, C.R. Jr Jack, J.E. Parisi, J.A. Lucas, R.C. Petersen, R. Rademakers, Characterization of frontotemporal dementia and/or 
amyotrophic lateral sclerosis associated with the GGGGCC repeat expansion in C9ORF72, Brain 135 (2012): 765-783.

[81] J. Yan, H.X. Deng, N. Siddique, F. Fecto, W. Chen, Y. Yang, E. Liu, S. Donkervoort, J.G. Zheng, Y. Shi, K.B. Ahmeti, B. Brooks, W.K. Engel, T. Siddique, Frameshift and novel mutations in FUS in familial amyotrophic lateral sclerosis and ALS/dementia, Neurology 75 (2010): 807-814.

[82] N. Chan, C. Le, P. Shieh, T. Mozaffar, M. Khare, J. Bronstein, V. Kimonis, Valosin-containing protein mutation and Parkinson's disease, Parkinsonism Relat. Disord. 18 (2012): 107-109.

[83] P.R. Jarman, N.W. Wood, M.T. Davis, P.V. Davis, K.P. Bhatia, C.D. Marsden, M.B. Davis, Hereditary geniospasm: linkage to chromosome 9q13-q21 and evidence for genetic heterogeneity, Am. J. Hum. Genet. 61 (1997): 928-933.

[84] A. Macerollo, T.A. Saifee, P. Kassavetis, G. Pilurzi, S.A. Schneider, M.J. Edwards, K.P. Bhatia, Abnormalities of masseteric inhibitory reflex in hereditary geniospasm: evidence for a brainstem myoclonus. Mov. Disord. Clin. Pract. 2 (2015): 49-52.

[85] A.F. Gao, A. Faust-Socher, M. Al-Murshed, M.R. Del Bigio, A.E. Lang, D.G. Munoz, Progressive ataxia and palatal tremor: two autopsy cases of a novel tauopathy, Mov. Disord. 32 (2017): 14651473.

[86] M. Brenner, A.B. Johnson, O. Boespflug-Tanguy, D. Rodriguez, J.E. Goldman, A. Messing, Mutation in GFAP, encoding glial fibrillary acidic protein, are associated with Alexander disease, Nat. Genet. 27 (2001): 117-120.

[87] E. Pretegiani, F. Rosini, P. Federighi, A. Cerase, M.T. Dotti, A. Rufa, Pendular nystagmus, palatal tremor and progressive ataxia in GM2-gangliosidosis, Eur. J. Neurol. 22 (2015): e67-e69.

[88] M. Rossi, M. Cesarini, E.M. Gatto, A. Cammarota, M. Merello, A treatable rare cause of progressive ataxia and palatal tremor, Tremor Other Hyperkinet. Mov. (NY) 8 (2018): 538.

[89] N. Nicastro, E. Ranza, S.E. Antonarakis, J. Horvath, Pure progressive ataxia and palatal tremor (PAPT) associated with a new polymerase gamma (POLG) mutation, Cerebellum 15 (2016): 829-831.

[90] D. Thyagarajan, T. Chataway, R. Li, W.P. Gai, M. Brenner, Dominantly-inherited adult-onset leukodystrophy with palatal tremor caused by a mutation in the glial fibrillary acidic protein gene, Mov. Disord. 19 (2004): 1244-1248. 
[91] T. Yoshida, M. Nakagawa, Clinical aspects and pathology of Alexander disease, and morphological and functional alteration of astrocytes induced by GFAP mutation, Neuropathology 32 (2012): 440446.

[92] M. Namekawa, Y. Takiyama, J. Honda, H. Schimazaki, K. Sakoe, I. Nakano, Adult-onset Alexander disease with typical "tadpole" brainstem atrophy and unusual bilateral basal ganglia involvement: a case report and review of the literature, BMC Neurol. 10 (2010): 21.

[93] T. Lo Giudice, F. Lombardi, F.M. Santorelli, T. Kawarai, A. Orlacchio, Hereditary spastic paraplegia: clinical-genetic characteristics and evolving molecular mechanisms, Exp. Neurol. 261 (2014): 518539.

[94] R. Erro, C. Cordivari, K.P. Bhatia, SPG31 presenting with orthostatic tremor, Eur. J. Neurol. 21 (2014): e34-e35.

[95] M. Picillo, R. Erro, R.P. Munhoz, A. Fasano, When shaking during standing points to hereditary spastic paraplegias, Parkinsonism Relat. Disord. 46 (2018): 92-94.

[96] A. Molloy, O. Kimmich, J. Martindale, H. Moore, M. Hutchinson, S. O'Riordan, A novel CACNA1A mutation associated with adult-onset, paroxysmal head tremor, Mov. Disord. 28 (2013): 842-843.

[97] E. Bernard, E. Broussolle, S. Thobois, Postural tremor as first symptom of Huntington disease, Rev. Neurol. (Paris) 166 (2010): 841-843.

[98] T. Nguyen, T.C. Danaila, G.L. Defer, Isolated postural tremor revealing Huntington's disease [abstract]. Mov. Disord. 27 Suppl. 1 (2012): 172.

[99] T. Konno, O.A. Ross, H.A.G. Teive, J. Sławek, D.W. Dickson, Z.K. Wszolek, DCTN1-related neurodegeneration: Perry syndrome and beyond, Parkinsonism Relat. Disord. 41 (2017): 14-24.

[100] A. Batla, X.Y. Tai, L. Schottlaender, R. Erro, B. Balint, K.P. Bhatia, Deconstructing Fahr's disease/syndrome of brain calcification in the era of new genes, Parkinsonism Relat. Disord. 37 (2017): 1-10.

[101] C. Sundal, S. Fujioka, J.A. Van Gerpen, C. Wider, A.M. Nicholson, M. Baker, E.A. Shuster, J. Aasly, S. Spina, B. Ghetti, S. Roeber, J. Garbern, A. Tselis, R.H. Swerdlow, B.B. Miller, A. BorjessonHanson, R.J. Uitti, O.A. Ross, A.J. Stoessl, R. Rademakers, K.A. Josephs, D.W. Dickson, D. 
Broderick, Z.K. Wszolek, Parkinsonian features in hereditary diffuse leukoencephalopathy with spheroids (HDLS) and CSF1R mutations, Parkinsonism Relat. Disord. 19 (2013): 869-877.

[102] T.A. Saifee, I. Pareés, P. Kassavetis, D. Kaski, A.M. Bronstein, J.C. Rothwell, A. Sadnicka, M.P. Lunn, H. Manji, J.T. Teo, K.P. Bhatia, M.M. Reilly, M.J. Edwards, Tremor in Charcot-Marie-Tooth disease: no evidence of cerebellar dysfunction, Clin. Neurophysiol. 126 (2015): 1817-1824.

[103] M.T. Lorincz, Neurologic Wilson’s disease, Ann. NY Acad. Sci. 1184 (2010): 173-187.

[104] A. Członkowska, T. Litwin, G. Chabik, Wilson disease: neurologic features, Handb. Clin. Neurol. 142 (2017): 101-119.

[105] H. Topaloglu, K Gücüyener, C Orkun, Y Renda, Tremor of tongue and dysarthria as the sole manifestation of Wilson's disease, Clin. Neurol. Neurosurg. 92 (1990): 295-296.

[106] H. El Otmani, T. Benhsain, Z. Abdulhakeem, B. El Moutawakil, M.A. Rafai, Ondulating tongue revealing Wilson's disease, Mov. Dis. Clin. Pract. 6 (2019): 605-607.

[107] S.A. Schneider, K.P. Bhatia, J. Hardy, Complicated recessive dystonia parkinsonism syndromes, Mov. Disord. 24 (2009): 490-499.

[108] E. Lohmann, M. Periquet, V. Bonifati, N.W. Wood, G. De Michele, A.M. Bonnet, V. Fraix, E. Broussolle, M.W. Horstink, M. Vidailhet, P. Verpillat, T. Gasser, D. Nicholl, H. Teive, S. Raskin, O. Rascol, A. Destée, M. Ruberg, F. Gasparini, G. Meco, Y. Agid, A. Durr, A. Brice; French Parkinson's Disease Genetics Study Group; European Consortium on Genetic Susceptibility in Parkinson's Disease, How much phenotypic variation can be attributed to parkin genotype?, Ann. Neurol. 54 (2003); 54: 176-185.

[109] S. Edvardson, Y. Cinnamon, A. Ta-Shma, A. Shaag, Y.I. Yim, S. Zenvirt, C. Jalas, S. Lesage, A. Brice, A. Taraboulos, K.H. Kaestner, L.E. Greene, O. Elpeleg, A deleterious mutation in DNAJC6 encoding the neuronal-specific clathrin-uncoating co-chaperone auxilin, is associated with juvenile parkinsonism, PLoS One 7 (2012): e36458.

[110] C.E. Krebs, S. Karkheiran, J.C. Powell, M. Cao, V. Makarov, H. Darvish, G. Di Paolo, R.H. Walker, G.A. Shahidi, J.D. Buxbaum, P. De Camilli, Z. Yue, C. Paisán-Ruiz, The Sac1 domain of SYNJ1 identified mutated in a family with early-onset progressive Parkinsonism with generalized seizures, Hum. Mutat. 34 (2013): 1200-1207. 
[111] M. Quadri, M. Fang, M. Picillo, S. Olgiati, G.J. Breedveld, J. Graafland, B. Wu, F. Xu, R. Erro, M. Amboni, S. Pappatà, M. Quarantelli, G. Annesi, A. Quattrone, H.F. Chien, E.R. Barbosa; International Parkinsonism Genetics Network, B.A. Oostra, P. Barone, J. Wang, V. Bonifati, Mutation in the SYNJ1 gene associated with autosomal recessive, early-onset Parkinsonism, Hum. Mutat. 34 (2013): 1208-1215.

[112] J. Ng, J. Zhen, E. Meyer, K. Erreger, Y. Li, N. Kakar, J. Ahmad, H. Thiele, C. Kubisch, N.L. Rider, D.H. Morton, K.A. Strauss, E.G. Puffenberger, D. D'Agnano, Y. Anikster, C. Carducci, K. Hyland, M. Rotstein, V. Leuzzi, G. Borck, M.E. Reith, M.A. Kurian, Dopamine transporter deficiency syndrome: phenotypic spectrum from infancy to adulthood, Brain 137 (2014): 1107-1119.

[113] E. Mulroy, B. Balint, E. Menozzi, A. Latorre, K.P. Bhatia, Benign tremulous parkinsonism of the young - consider Parkin, Parkinsonism Relat. Disord. 65 (2019): 270-271.

[114] S.J. Hayflick, M.A. Kurian, P. Hogarth, Neurodegeneration with brain iron accumulation, Handb. Clin. Neurol. 147 (2018): 293-305.

[115] B. Zhou, S.K. Westaway, B. Levinson, M.A. Johnson, J. Gitschier, S.J. Hayflick, A novel pantothenate kinase gene (PANK2) is defective in Hallervorden-Spatz syndrome, Nature Genetics 28 (2001): 345-349.

[116] S. Yamashita, Y. Maeda, H. Ohmori, Y. Uchida, T. Hirano, K. Yonemura, E. Uyama, M. Uchino, Pantothenate kinase-associated neurodegeneration initially presenting as postural tremor alone in a Japanese family with homozygous N245S substitutions in the pantothenate kinase gene, J. Neurol. Sci. 225 (2004): 129-133.

[117] A. Aggarwal, S.A. Schneider, H. Houlden, M. Silverdale, R. Paudel, C. Paisan-Ruiz, S. Desai, M. Munshi, D. Sanghvi, J. Hardy, K.P. Bhatia, M. Bhatt, Indian-subcontinent NBIA: unusual phenotypes, novel PANK2 mutations, and undetermined genetic forms. Mov. Disord. 25 (2010): 1424-1231.

[118] W.T. Yoon, W.Y. Lee, H.Y. Shin, S.T. Lee, C.S. Ki, Novel PANK2 gene mutations in Korean patient with pantothenate kinase-associated neurodegeneration presenting unilateral dystonic tremor, Mov. Disord. 25 (2010): 245-247.

[119] M. Rohani, G. Shahidi, A. Alavi, A.E. Lang, N. Yousefi, S. Razme, A. Fasano, Tremor-dominant pantothenate kinase-associated neurodegeneration, Mov. Dis. Clin. Pract. 4 (2017): 772-774. 
[120] S. Karkheiran, G.A. Shahidi, R.H. Walker, C. Paisán-Ruiz, PLA2G6-associated dystoniaparkinsonism: case report and literature review, Tremor Other Hyperkinet. Mov. (NY) 5 (2015): 317.

[121] H. Miyajima, Y. Takahashi, S. Kono, Aceruloplasminemia, an inherited disorder of iron metabolism, Biometals 16 (2003): 205e213.

[122] L.H.P. Vroegindeweij, J.G. Langendonk, M. Langeveld, M. Hoogendoorn, A.J.A. Kievit, D. Di Raimondo, J.H.P. Wilson, A.J.W. Boon, New insights in the neurological phenotype of aceruloplasminemia in Caucasian patients, Parkinsonism Relat. Disord. 36 (2017): 33-40.

[123] M. Hartig, H. Prokisch, T. Meitinger, T. Klopstock, Mitochondrial membrane protein-associated neurodegeneration (MPAN), Int. Rev. Neurobiol. 110 (2013): 73-84.

[124] R. Horvath, E. Holinski-Feder, V.C. Neeve, A. Pyle, H. Griffin, D. Ashok, C. Foley, G. Hudson, B. Rautenstrauss, G. Nürnberg, P. Nürnberg, J. Kortler, B. Neitzel, I. Bässmann, T. Rahman, B. Keavney, J. Loughlin, S. Hambleton, B. Schoser, H. Lochmüller, M. Santibanez-Koref, P.F. Chinnery, A new phenotype of brain iron accumulation with dystonia, optic atrophy, and peripheral neuropathy, Mov. Disord. 27 (2012): 789-793.

[125] A. Gregory, M. Lotia, S.Y. Jeong, R. Fox, D. Zhen, L. Sanford, J. Hamada, A. Jahic, C. Beetz, A. Freed, M.A. Kurian, T. Cullup, M.C.M. van der Weijden, V. Nguyen, N. Setthavongsack, D. Garcia, V. Krajbich, T. Pham, R. Woltjer, B.P. George, K.Q. Minks, A.R. Paciorkowski, P. Hogarth, J. Jankovic, S.J. Hayflick, Autosomal dominant mitochondrial membrane protein-associated neurodegeneration (MPAN), Mol. Genet. Genomic Med. 7 (2019): e00736.

[126] F. Sedel, J.M. Saudubray, E. Roze, Y. Agid, M. Vidailhet, Movement disorders and inborn errors of metabolism in adults: a diagnostic approach, J. Inherit. Metab. Dis. 31 (2008): 308-318.

[127] H.S. Singer, J.W. Mink, D.L. Gilbert, J. Jankovic, Inherited metabolic disorders with associated movement abnormalities, in: H.S. Singer, J.W. Mink, D.L. Gilbert, J. Jankovic (Eds.), Movement Disorders in Childhood 2nd Edition, Elsevier Inc., USA, 2016, pp. 337-406.

[128] S. Külkens, I. Harting, S. Sauer, J. Zschocke, G.F. Hoffmann, S. Gruber, O.A. Bodamer, S. Kölker, Late-onset neurologic disease in glutaryl-CoA dehydrogenase deficiency, Neurology 64 (2005): 21422144. 
[129] I. Rubio-Agusti, M. Carecchio, K.P. Bhatia, M. Kojovic, I. Parees, H.S. Chandrashekar, E.J. Footitt, D. Burke, M.J. Edwards, R.H. Lachmann, E. Murphy, Movement disorders in adult patients with classical galactosemia, Mov. Disord. 28 (2013): 804-810.

[130] M. Beaudin, C.J. Klein, G.A. Rouleau, N, Systematic review of autosomal recessive ataxias and proposal for a classification, Cerebellum Ataxias 4 (2017): 3.

[131] J.G. Hou, J. Jankovic, Movement disorders in Friedreich's ataxia, Neurol. Sci. 206 (2003): 59-64.

[132] A. Méneret, Y. Ahmar-Beaugendre, G. Rieunier, N. Mahlaoui, B. Gaymard, E. Apartis, C. Tranchant, S. Rivaud-Péchoux, B. Degos, B. Benyahia, F. Suarez, T. Maisonobe, M. Koenig, A. Durr, M.H. Stern, C. Dubois d'Enghien, A. Fischer, M. Vidailhet, D. Stoppa-Lyonnet, D. Grabli, M. Anheim, The pleiotropic movement disorders phenotype of adult ataxia-telangiectasia, Neurology 83 (2014): 1087 1095.

[133] A. Levy, A.E. Lang, Ataxia-telangiectasia: a review of movement disorders, clinical features, and genotype correlations, Mov. Disord. 33 (2018): 1238-1247.

[134] M. Anheim, B. Monga, M. Fleury, P. Charles, C. Barbot, M. Salih, J.P. Delaunoy, M. Fritsch, L. Arning, M. Synofzik, L. Schöls, J. Sequeiros, C. Goizet, C. Marelli, I. Le Ber, J. Koht, J. Gazulla, J. De Bleecker, M. Mukhtar, N. Drouot, L. Ali-Pacha, T. Benhassine, M. Chbicheb, A. M'Zahem, A. Hamri, B. Chabrol, J. Pouget, R. Murphy, M. Watanabe, P. Coutinho, M. Tazir, A. Durr, A. Brice, C. Tranchant, M. Koenig, Ataxia with oculomotor apraxia type 2: clinical, biological and genotype/phenotype correlation study of a cohort of 90 patients, Brain 132 (2009): 2688-2698.

[135] M. Stamelou, K. Tuschl, W.K. Chong, A.K. Burroughs, P.B. Mills, K.P. Bhatia, P.T. Clayton, Dystonia with brain manganese accumulation resulting from SLC30A10 mutations: a new treatable disorder, Mov. Disord. 27 (2012): 1317-1322.

[136] K. Tuschl, E. Meyer, L.E. Valdivia, N. Zhao, C. Dadswell, A. Abdul-Sada, C.Y. Hung, M.A. Simpson, W.K. Chong, T.S. Jacques, R.L. Woltjer, S. Eaton, A. Gregory, L. Sanford, E. Kara, H. Houlden, S.M. Cuno, H. Prokisch, L. Valletta, V. Tiranti, R. Younis, E.R. Maher, J. Spencer, A. Straatman-Iwanowska, P. Gissen, L.A. Selim, G. Pintos-Morell, W. Coroleu-Lletget, S.S. Mohammad, S. Yoganathan, R.C. Dale, M. Thomas, J. Rihel, O.A. Bodamer, C.A. Enns, S.J. Hayflick, P.T. 
Clayton, P.B. Mills, M.A. Kurian, S.W. Wilson, Mutations in SLC39A14 disrupt manganese homeostasis and cause childhood-onset parkinsonism-dystonia, Nat. Commun. 7 (2016): 11601.

[137] E. Apartis, A. Blancher, W.G. Meissner, L. Guyant-Maréchal, D. Maltête, T. De Broucker, A.P. Legrand, H. Bouzenada, H.T. Thanh, M. Sallansonnet-Froment, A. Wang, F. Tison, C. Roué-Jagot, F. Sedel, P. Charles, S. Whalen, D. Héron, S. Thobois, A. Poisson, G. Lesca, A.M. Ouvrard-Hernandez, V. Fraix, S. Palfi, M.O. Habert, B. Gaymard, J.C. Dussaule, P. Pollak, M. Vidailhet, A. Durr, J.C. Barbot, V. Gourlet, A. Brice, M. Anheim, FXTAS: new insights and the need for revised diagnostic criteria, Neurology 79 (2012): 1898-1907.

[138] R.J. Hagerman, P. Hagerman, Fragile X-associated tremor/ataxia syndrome - features, mechanisms and management, Nat. Rev. Neurol. 12 (2016): 403-412.

[139] D. Hall, K. Todorova-Koteva, S. Pandya, B. Bernard, B. Ouyang, M. Walsh, T. Pounardjian, C. Deburghraeve, L. Zhou, M. Losh, M. Leehey, E. Berry-Kravis, Neurological and endocrine phenotypes of fragile X carrier women, Clin. Genet. 89 (2016): 60-67.

[140] D.C. Bragg, K. Mangkalaphiban, C.A. Vaine, N.J. Kulkarni, D. Shin, R. Yadav, J. Dhakal, M.L. Ton, A. Cheng, C.T. Russo, M. Ang, P. Acuña, C. Go, T.N. Franceour, T. Multhaupt-Buell, N. Ito, U. Müller, W.T. Hendriks, X.O. Breakefield, N. Sharma, L.J. Ozelius, Disease onset in X-linked dystonia-parkinsonism correlates with expansion of a hexameric repeat within an SVA retrotransposon in TAF1, Proc. Natl. Acad. Sci. USA 114 (2017): E11020-E11028.

[141] V.G. Evidente, J. Advincula, R. Esteban, P. Pasco, J.A. Alfon, F.F. Natividad, J. Cuanang, A.S. Luis, K. Gwinn-Hardy, J. Hardy, D. Hernandez, A. Singleton, Phenomenology of "Lubag" or X-linked dystonia-parkinsonism, Mov. Disord. 17 (2002): 1271-1277.

[142] V.G. Evidente, D. Nolte, S. Niemann, J. Advincula, M.C. Mayo, F.F. Natividad, U. Müller, Phenotypic and molecular analyses of X-linked dystonia-parkinsonism ("Lubag") in women, Arch. Neurol. 61 (2004): 1956-1959.

[143] G.R. Wilson, J.C. Sim, C. McLean, M. Giannandrea, C.A. Galea, J.R. Riseley, S.E. Stephenson, E. Fitzpatrick, S.A. Haas, K. Pope, K.J. Hogan, R.G. Gregg, C.J. Bromhead, D.S. Wargowski, C.H. Lawrence, P.A. James, A. Churchyard, Y. Gao, D.G. Phelan, G. Gillies, N. Salce, L. Stanford, A.P. Marsh, M.L. Mignogna, S.J. Hayflick, R.J. Leventer, M.B. Delatycki, G.D. Mellick, V.M. Kalscheuer, 
P. D'Adamo, M. Bahlo, D.J. Amor, P.J. Lockhart, Mutations in RAB39B cause X-linked intellectual disability and early-onset Parkinson disease with $\alpha$-synuclein pathology, Am. J. Hum. Genet. 95 (2014): 729-735.

[144] T. Temudo, M. Santos, E. Ramos, K. Dias, J.P. Vieira, A. Moreira, E. Calado, I. Carrilho, G. Oliveira, A. Levy, C. Barbot, M. Fonseca, A. Cabral, P. Cabral, J. Monteiro, L. Borges, R. Gomes, G. Mira, S.A. Pereira, M. Santos, A. Fernandes, J.T. Epplen, J. Sequeiros, P. Maciel, Rett syndrome with and without detected MECP2 mutations: an attempt to redefine phenotypes, Brain. Dev. 33 (2011): 69-76.

[145] R. Hanajima, Y. Terao, S. Nakatani-Enomoto, M. Hamada, A. Yugeta, H. Matsumoto, T. Yamamoto, S. Tsuji, Y. Ugawa, Postural tremor in X-linked spinal and bulbar muscular atrophy, Mov. Disord. 24 (2009): 2063-2069.

[146] S.J. Hayflick, M.C. Kruer, A. Gregory, T.B. Haack, M.A. Kurian, H.H. Houlden, J. Anderson, N. Boddaert, L. Sanford, S.I. Harik, V.H. Dandu, N. Nardocci, G. Zorzi, T. Dunaway, M. Tarnopolsky, S. Skinner, K.R. Holden, S. Frucht, E. Hanspal, C. Schrander-Stumpel, C. Mignot, D. Héron, D.E. Saunders, M. Kaminska, J.P. Lin, K. Lascelles, S.M. Cuno, E. Meyer, B. Garavaglia, K. Bhatia, R. de Silva, S. Crisp, P. Lunt, M. Carey, J. Hardy, T. Meitinger, H. Prokisch, P. Hogarth, $\beta$-Propeller protein-associated neurodegeneration: a new X-linked dominant disorder with brain iron accumulation, Brain 136 (2013): 1708-1717.

[147] Y.B. Hong, J.M. Park, J.S. Yu, D.H. Yoo, D.E. Nam, H.J. Park, J.S. Lee, S.H. Hwang, K.W. Chung, B.O. Choi, Clinical characterization and genetic analysis of Korean patients with X-linked CharcotMarie-Tooth disease type 1, J. Peripher. Nerv. Syst. 22 (2017): 172-181.

[148] S. Rahman, W.C. Copeland, POLG-related disorders and their neurological manifestations, Nat. Rev. Neurol. 15 (2019): 40-52.

[149] S.R. Schreglmann, F. Riederer, M. Galovic, C. Ganos, G. Kägi, D. Waldvogel, Z. Jaunmuktane, A. Schaller, U. Hidding, E. Krasemann, L. Michels, C.R. Baumann, K. Bhatia, H.H. Jung HH, Movement disorders in genetically confirmed mitochondrial disease and the putative role of the cerebellum, Mov. Disord. 33 (2018): 146-155.

[150] M. Milone, B.T. Klassen, M.L. Landsverk, R.H. Haas, J.J. Wong, Orthostatic tremor, progressive external ophthalmoplegia, and Twinkle, JAMA Neurol. 70 (2013): 1429-1431. 
[151] R. Ghaoui, C.M. Sue, Movement disorders in mitochondrial disease, J. Neurol. 265 (2018): 12301240.

[152] C. Tranchant, M. Anheim, Movement disorders in mitochondrial diseases, Rev. Neurol. (Paris) 172 (2016): 524-529.

[153] D.S. Hong, A.L. Reiss, Cognitive and neurological aspects of sex chromosome aneuploidies, Lancet Neurol. 13 (2014): 306-318.

[154] T.L. Harlow, P. Gonzalez-Alegre, High prevalence of reported tremor in Klinefelter syndrome, Parkinsonism Relat. Disord. 15 (2009): 393-395.

[155] M. Koegl-Wallner, P. Katschnig-Winter, T. Pendl, B. Melisch, M. Trummer, E. Holl, U. Werner, R. Schmidt, P. Schwingenschuh, Tremor associated with Klinefelter syndrome--a case series and review of the literature, Parkinsonism Relat. Disord. 20 (2014): 323-327.

[156] M.Z. Bardsley, K. Kowal, C. Levy, A. Gosek, N. Ayari, N. Tartaglia, N. Lahlou, B. Winder, S. Grimes, J.L. Ross, 47,XYY syndrome: clinical phenotype and timing of ascertainment, J. Pediatr. 163 (2013): 1085-1094.

[157] N. Tartaglia, N. Ayari, S. Howell, C. D'Epagnier, P. Zeitler, 48,XXYY, 48,XXXY and 49,XXXXY syndromes: not just variants of Klinefelter syndrome, Acta Paediatr. 100 (2011): 851-860.

[158] N. Tartaglia, S. Davis, A. Hench, S. Nimishakavi, R. Beauregard, A. Reynolds, L. Fenton, L. Albrecht, J. Ross, J. Visootsak, R. Hansen, R. Hagerman, A new look at XXYY syndrome: medical and psychological features, Am. J. Med. Genet. 146A (2008): 1509-1522.

[159] N. Tartaglia, M. Borodyanskaya, D.A. Hall, Tremor in 48,XXYY syndrome, Mov. Disord. 24 (2009): 2001-2007.

[160] E. Boot, A.S. Bassett, C. Marras, 22q11.2 deletion syndrome-associated Parkinson's disease, Mov. Dis. Clin. Pract. 6 (2018): 11-16.

[161] E.K. Tan, Chromosomal deletion at 22q11.2 and Parkinson's disease, Lancet Neurol. 15 (2016): 538540.

[162] M. Carecchio, N.E. Mencacci, Emerging monogenic complex hyperkinetic disorders, Curr. Neurol. Neurosci. Rep. 17 (2017): 97. 
[163] C. Reale, C. Panteghini, M. Carecchio, B. Garavaglia, The relevance of gene panels in movement disorders diagnosis: a lab perspective, Eur. J. Paediatr. Neurol. 22 (2018): 285-291.

[164] S. Yohe, B. Thyagarajan, Review of clinical next-generation sequencing, Arch. Pathol. Lab. Med. 141 (2017): $1544-1557$. 
Table 1. Summary of loci and genes which have hitherto been associated with essential tremor in chronological order

\begin{tabular}{|c|c|c|c|c|c|c|}
\hline Locus/Gene & Chromosome & Gene product & Study type $^{\wedge}$ & Study population & Criteria to define ET in the study & Reference \\
\hline ETM1 & $3 q 13$ & - (see $D R D 3)$ & GWS+LA & $\begin{array}{l}16 \text { small Icelandic families }(75 \\
\text { ET) }\end{array}$ & $\begin{array}{l}\text { TRIG criteria for definite ET.[2,30] Bilateral postural tremor, with or } \\
\text { without kinetic tremor, of the hands or forearms which was visible and } \\
\text { persistent and had lasted for at least five years. Absence of other } \\
\text { neurological signs, including dystonia. }\end{array}$ & [6] \\
\hline ETM2 & 2p22-p25 & - (see $H S 1 B P 3)$ & GWS+LA & $\begin{array}{l}1 \text { large Czech/American family } \\
(18 \text { ET) }\end{array}$ & $\begin{array}{l}\text { Bilateral postural tremor, with or without kinetic tremor, that was } \\
\text { visible and persistent in the hands or forearms and had moderate ( } 2 \text { - to } \\
4-\mathrm{cm} \text { excursion) or coarse }(>4-\mathrm{cm} \text { excursion) tremor amplitudes and } \\
\text { slow tremor frequency }(4 \text { to } 10 \mathrm{~Hz}) \text {. }\end{array}$ & [7] \\
\hline MTHFR & $1 \mathrm{p} 36.22$ & $\begin{array}{l}\text { Methylenetetrahydrofolate } \\
\text { reductase }\end{array}$ & $\mathrm{CG}+\mathrm{CCGAS}$ & $\begin{array}{l}158 \text { unrelated Caucasian ET } \\
\text { and } 246 \text { unrelated Caucasian } \\
\text { HC }\end{array}$ & Criteria for ET according to [31] and [32]. & [8] \\
\hline HS1-BP3 & $2 \mathrm{p} 24.1$ & $\begin{array}{l}\text { Hematopoietic lineage cell specific } \\
\text { protein binding protein } 3\end{array}$ & FM & 2 unrelated US families & $\begin{array}{l}\text { Definite or probable ET according to [30]. Bilateral postural tremor of } \\
\text { the hands or forearms which was visible and persistent with at least 1- } \\
\text { to } 2 \text {-cm excursions in at least one arm. Absence of parkinsonism, } \\
\text { dystonia, myoclonus, peripheral neuropathy, or restless legs syndrome. }\end{array}$ & [9] \\
\hline ETM3 & $6 \mathrm{p} 23$ & - & GWS+LA & $\begin{array}{l}7 \text { North American families ( } 65 \\
\text { ET) }\end{array}$ & $\begin{array}{l}\text { Definite ET according to [33]. Bilateral tremor of minimum 5-year } \\
\text { duration and severity grades three or four with secondary causes of } \\
\text { tremor excluded. }\end{array}$ & [10] \\
\hline DRD3 & $3 \mathrm{q} 13.31$ & Dopamine receptor D3 & CG+LA & 30 unrelated ET French families & $\begin{array}{l}\text { Definite or probable familial ET diagnosed according to the guidelines } \\
\text { of the } 1998 \text { MDS Consensus Statement.[2] }\end{array}$ & [11] \\
\hline LINGO1 & $15 \mathrm{q} 24.3$ & $\begin{array}{l}\text { Leucine-rich repeat and Ig domain } \\
\text { containing } 1\end{array}$ & GWAS & $\begin{array}{l}\text { First stage: } 452 \text { Icelandic ET } \\
\text { and 14,394 HC. } \\
\text { Second stage: } 301 \text { ET and 1,419 } \\
\text { HC of European (Austria, } \\
\text { Germany, Iceland) or US } \\
\text { origin. }\end{array}$ & $\begin{array}{l}\text { Icelandic families: TRIG criteria for definite ET,[2, 30] i.e. bilateral } \\
\text { postural tremor, with or without kinetic tremor, of hands or forearms, } \\
\text { visible and persistent and lasting for at least five years. Absence of } \\
\text { abnormal neurological signs, including dystonia and Parkinson's } \\
\text { disease. } \\
\text { Austrian families: ET diagnosed according to the guidelines of the } \\
1998 \text { MDS Consensus Statement.[2] Exclusion of subjects with the co- } \\
\text { occurrence of PD and ET. } \\
\text { German families: not specified. } \\
\text { American families: definite or probable ET according to the guidelines } \\
\text { of the } 1998 \text { MDS Consensus Statement[2] and TRIG criteria.[30] }\end{array}$ & [12] \\
\hline MAPT & $17 \mathrm{q} 21.31$ & Microtubule-associated protein tau & $\mathrm{CG}+\mathrm{CCGAS}$ & $\begin{array}{l}356 \text { Caucasian ET and } 409 \\
\text { Caucasian HC from North } \\
\text { America }\end{array}$ & Not specified. & [13] \\
\hline$S L C 1 A 2$ & $11 \mathrm{p} 13$ & Excitatory amino acid transporter 2 & GWAS & $\begin{array}{l}\text { First stage: } 436 \mathrm{ET} \text { and } 928 \mathrm{HC} \\
\text { of German origin. } \\
\text { Second stage: } 554 \mathrm{ET} \text { and } 609 \\
\text { HC of European (Germany, } \\
\text { Denmark, Austria) origin. }\end{array}$ & $\begin{array}{l}\text { ET diagnosed according to the guidelines of the } 1998 \text { MDS Consensus } \\
\text { Statement.[2] }\end{array}$ & [14] \\
\hline $\begin{array}{l}\text { FUS } \\
\text { (ETM4) }\end{array}$ & $16 \mathrm{p} 11.2$ & $\begin{array}{l}\text { Fused in sarcoma/translated in } \\
\text { liposarcoma }\end{array}$ & WES & $\begin{array}{l}\text { First stage: } 1 \text { large French- } \\
\text { Canadian family ( } 23 \text { ET, } 12 \\
\text { HC). } \\
\text { Second stage: } 270 \text { ET. }\end{array}$ & $\begin{array}{l}\text { Definite ET according to the guidelines of the } 1998 \text { MDS Consensus } \\
\text { Statement.[2] }\end{array}$ & [15] \\
\hline
\end{tabular}




\begin{tabular}{|c|c|c|c|c|c|c|}
\hline HTRA2 & $2 \mathrm{p} 13.1$ & Mitochondrial serine protease & WES & $\begin{array}{l}\text { First stage: } 1 \text { large Turkish } \\
\text { family (11 ET, } 5 \mathrm{ET}+\mathrm{PD}, 8 \\
\text { HC). } \\
\text { Second stage: } 25 \text { other Turkish } \\
\text { ET families (each including } \\
\text { multiple ET) and } 364 \text { HC. }\end{array}$ & $\begin{array}{l}\text { ET diagnosed according to the guidelines of the } 1998 \text { MDS Consensus } \\
\text { Statement.[2] Five subjects were reported to have co-existence of PD } \\
\text { and ET. }\end{array}$ & [16] \\
\hline $\begin{array}{l}\text { TENM4 } \\
\text { (ETM5) }\end{array}$ & $11 \mathrm{q} 14.1$ & Teneurin transmembrane protein 4 & WES & $\begin{array}{l}\text { First stage: } 3 \text { Spanish families } \\
\text { (23 ET, } 12 \text { HC). } \\
\text { Second stage: } 299 \text { unrelated } \\
\text { Spanish ET. }\end{array}$ & $\begin{array}{l}\text { ET diagnosed according to the guidelines of the } 1998 \text { MDS Consensus } \\
\text { Statement.[2] }\end{array}$ & [17] \\
\hline TREM2 & $6 \mathrm{p} 21.1$ & $\begin{array}{l}\text { Triggering receptor expressed on } \\
\text { myeloid cells } 2\end{array}$ & $\mathrm{CG}+\mathrm{CCGAS}$ & $\begin{array}{l}\text { First stage: } 456 \text { ET and } 2,715 \\
\text { HC of Spanish origin. } \\
\text { Second stage: } 897 \text { ET and } 1,449 \\
\text { HC from Germany, Italy, } \\
\text { America, and Taiwan. } \\
\text { Third stage: } 14,777 \text { HC from } \\
\text { Spain, Italy, Germany, North } \\
\text { America, China. }\end{array}$ & $\begin{array}{l}\text { Definite or probable ET diagnosed according to the guidelines of the } \\
1998 \text { MDS Consensus Statement.[2] }\end{array}$ & [18] \\
\hline SORT1 & $1 \mathrm{p} 13.3$ & Sortilin 1 & WES & $\begin{array}{l}1 \text { small Spanish family (2 ET, } 4 \\
\mathrm{HC})\end{array}$ & $\begin{array}{l}\text { ET according to the guidelines of the } 1998 \text { MDS Consensus } \\
\text { Statement[2] and the WHIGET clinical criteria.[34] }\end{array}$ & [19] \\
\hline$S C N 4 A$ & $17 \mathrm{q} 23.3$ & $\begin{array}{l}\text { Voltage-gated sodium channel, } \\
\text { type } 4 \text {, alpha subunit }\end{array}$ & WES & $\begin{array}{l}\text { First stage: } 1 \text { large Spanish } \\
\text { family with ET and epilepsy ( } 7 \\
\text { ET). } \\
\text { Second stage: } 76 \text { sporadic and } \\
25 \text { familial ET. }\end{array}$ & $\begin{array}{l}\text { Not specified, but apparently not isolated tremor syndrome in the index } \\
\text { family (one subject had postural tremor of both hands, head tremor, } \\
\text { mild resting tremor and a mild ataxic gait; one subject had myoclonus, } \\
\text { postural tremor of both hands and head tremor). } \\
\text { In the second stage, ET according to the guidelines of the } 1998 \text { MDS } \\
\text { Consensus Statement[2] and the WHIGET criteria clinical criteria were } \\
\text { comprehensively reviewed by the Consensus Statement of the } \\
\text { Movement Disorder Society on Tremor.[34] }\end{array}$ & [20] \\
\hline DNAJC13 & $3 \mathrm{q} 22.1$ & $\begin{array}{l}\text { DnaJ (Hsp40) homolog, subfamily } \\
\text { C, member } 13\end{array}$ & WES & $\begin{array}{l}\text { 571 ET of European descent } \\
\text { from North America }\end{array}$ & ET according to standard diagnostic criteria.[31] & [21] \\
\hline NOS3 & $7 \mathrm{q} 36.1$ & Nitric oxide synthase 3 & \multirow{4}{*}{ WES } & \multirow{3}{*}{$\begin{array}{l}37 \text { families with } 3 \text { to } 7 \text { ET } \\
\text { enrolled in the US }\end{array}$} & \multirow[t]{4}{*}{ ET according to the WHIGET criteria clinical criteria.[34] } & \multirow{4}{*}{ [22] } \\
\hline HAPLN4 & $19 \mathrm{p} 13.11$ & $\begin{array}{l}\text { Hyaluronan and proteoglycan link } \\
\text { protein } 4\end{array}$ & & & & \\
\hline USP46 & $4 \mathrm{q} 12$ & Ubiquitin-specific protease 46 & & & & \\
\hline KCNS2 & $8 \mathrm{q} 22.2$ & $\begin{array}{l}\text { Voltage-gated potassium channel } \\
\text { subunit Kv } 9.2\end{array}$ & & $\begin{array}{l}37 \text { families with } 3 \text { to } 7 \text { ET } \\
\text { enrolled in the US and } 95 \\
\text { unrelated ET }\end{array}$ & & \\
\hline STK32B & $4 \mathrm{p} 16.2$ & Serine/threonine kinase 32B & \multirow{3}{*}{ GWAS } & \multirow{3}{*}{$\begin{array}{l}\text { First stage: } 1,778 \text { ET and 5,376 } \\
\text { HC of North-Western European } \\
\text { ancestry. } \\
\text { Second stage: } 1,029 \text { ET and } \\
1,065 \text { HC of North-Western } \\
\text { European ancestry. }\end{array}$} & \multirow{3}{*}{$\begin{array}{l}\text { Definite or probable ET according to the guidelines of the } 1998 \text { MDS } \\
\text { Consensus Statement.[2] Postural and action tremor in the arms } \\
\text { exceeding the amplitude seen in enhanced physiological tremor and not } \\
\text { attributable to other causes (PD, dystonia, medication). }\end{array}$} & \multirow{3}{*}{ [23] } \\
\hline PPARGCIA & $4 \mathrm{p} 15.2$ & $\begin{array}{l}\text { Peroxisome proliferator-activated } \\
\text { receptor gamma coactivator 1- } \\
\text { alpha }\end{array}$ & & & & \\
\hline CTNNA3 & $10 \mathrm{q} 21.3$ & Catenin alpha 3 & & & & \\
\hline SCN11A & $3 \mathrm{p} 22.2$ & $\begin{array}{l}\text { Voltage-gated sodium channel } \\
\text { subunit alpha Nav1.9 }\end{array}$ & WES & $\begin{array}{l}1 \text { Chinese family with ET and } \\
\text { recurrent pain attacks }(8 \mathrm{ET})\end{array}$ & $\begin{array}{l}\text { Postural and action tremor localized to both upper limbs, without } \\
\text { tremor of the voice, head or other body parts. Rest tremor gradually } \\
\text { developing with age. }\end{array}$ & [24] \\
\hline$M C 1 R$ & $16 \mathrm{q} 24.3$ & Melanocortin 1 receptor & $\mathrm{CG}+\mathrm{CCGAS}$ & $\begin{array}{l}200 \text { Han Chinese ET and } 432 \\
\text { Han Chinese matched HC }\end{array}$ & $\begin{array}{l}\text { ET diagnosed according to the guidelines of the } 1998 \text { MDS Consensus } \\
\text { Statement[2] and [31]. }\end{array}$ & [25] \\
\hline$V D R$ & $12 q 13.11$ & Vitamin D receptor & $\mathrm{CG}+\mathrm{CCGAS}$ & $\begin{array}{l}239 \text { Caucasian sporadic ET and } \\
239 \text { unrelated matched } \\
\text { Caucasian HC }\end{array}$ & ET according to [30] and [33], no further details provided. & [26] \\
\hline
\end{tabular}




\begin{tabular}{|c|c|c|c|c|c|c|}
\hline$I L 1 B$ & $2 q 14.1$ & Interleukin 1-beta & CG+CCGAS & $\begin{array}{l}200 \text { Chinese ET and } 229 \\
\text { Chinese HC }\end{array}$ & $\begin{array}{l}\text { ET diagnosed according to the guidelines of the } 1998 \text { MDS Consensus } \\
\text { Statement.[2] Exclusion of parkinsonism, drug induced tremor, } \\
\text { cerebellar tremor, dystonia and tremors with hyperthyroidism. }\end{array}$ & [27] \\
\hline CACNAIG & $17 \mathrm{q} 21.33$ & $\begin{array}{l}\text { Calcium voltage-gated channel } \\
\text { subunit alpha } 1 \mathrm{G}\end{array}$ & \multirow{5}{*}{ WGS } & \multirow{5}{*}{$\begin{array}{l}8 \text { families ( } 31 \mathrm{ET}, 9 \mathrm{HC}) \\
\text { enrolled in the US }\end{array}$} & \multirow[t]{5}{*}{$\begin{array}{l}\text { Definite, probable, or possible ET according to the WHIGET } \\
\text { criteria.[34] }\end{array}$} & \multirow{5}{*}{ [28] } \\
\hline SLIT3 & $5 q 34-q 35$ & Slit guidance ligand 3 & & & & \\
\hline KARS & $16 \mathrm{q} 23.1$ & Lysyl-tRNA synthetase & & & & \\
\hline KIF5A & $12 \mathrm{q} 13.3$ & Kinesin family member $5 \mathrm{~A}$ & & & & \\
\hline NTRK1 & $1 \mathrm{q} 23.1$ & $\begin{array}{l}\text { Neurotrophic receptor tyrosine } \\
\text { kinase } 1\end{array}$ & & & & \\
\hline NOTCH2NLC & $1 \mathrm{q} 21.2$ & Notch $2 \mathrm{~N}$-terminal like $\mathrm{C}$ & $\begin{array}{l}\text { WES+LRS+RP- } \\
\text { PCR+GC-PCR }\end{array}$ & $\begin{array}{l}197 \text { ET pedigrees of Chinese } \\
\text { origin and } 1305 \text { Chinese HC }\end{array}$ & $\begin{array}{l}\text { ET diagnosed according to the guidelines of the } 1998 \text { MDS Consensus } \\
\text { Statement.[2] }\end{array}$ & [29] \\
\hline
\end{tabular}

Legend: CCGAS = case-control genetic association study; CG = candidate gene approach; ET = subjects with essential tremor; FM = fine mapping; GC-PCR = GC-rich polymerase chain reaction; GWAS = genome-wide association study; GWS = genome-wide scan; HC = healthy controls; LA = linkage analysis; LRS = long-read sequencing; MDS = Movement Disorder Society; PD = Parkinson's disease; RP-PCR = repeat-primed polymerase chain reaction; TR = targeted resequencing; TRIG = Tremor Investigation Group; WES = whole-exome sequencing; WGS = whole-genome sequencing; WHIGET = Washington Heights-

Inwood Genetic Study of Essential Tremor; ^ Original genetic study; replication studies not reported. 
Table 2. Main autosomal dominant inherited disorders which can present with tremor

\begin{tabular}{|c|c|c|c|c|c|c|c|}
\hline Tremor disorder & OMIM $^{\circledR}$ & Chromosome & Gene & Age of onset & Main phenotype $^{\wedge}$ & $\begin{array}{l}\text { Tremor } \\
\text { as rare } \\
\text { sign }^{\S}\end{array}$ & Laboratory and imaging findings \\
\hline \multicolumn{8}{|l|}{ Primary dystonias $^{1}$} \\
\hline $\begin{array}{l}\text { DYT-TOR1A } \\
\text { (DYT1) }\end{array}$ & 128100 & $9 \mathrm{q} 34.11$ & TORIA & $\begin{array}{l}\text { First-third } \\
\text { decade }\end{array}$ & $\begin{array}{l}\text { Early-onset mostly generalized dystonia, usually } \\
\text { involving lower limbs at onset. Isolated writing } \\
\text { tremor, head tremor, late onset rest tremor } \\
\text { reported. }\end{array}$ & & - \\
\hline $\begin{array}{l}\text { DYT/PARK-GCHI }{ }^{2} \\
\text { (DYT5a) }\end{array}$ & 128230 & $14 \mathrm{q} 22.2$ & GCH1 & $\begin{array}{l}\text { First-sixth } \\
\text { decade }\end{array}$ & $\begin{array}{l}\text { Progressive dopa-responsive dystonia with diurnal } \\
\text { fluctuation. Upper-limb postural and kinetic } \\
\text { tremor reported. Sometimes combined with } \\
\text { parkinsonism (including rest tremor), pyramidal } \\
\text { signs, scoliosis, anxiety, depression, obsessive-- } \\
\text { compulsive disorder, sleep disturbances. }\end{array}$ & & $\begin{array}{l}\text { Blood tests: high post-load phenylalanine. } \\
\text { CSF: low neopterin, biopterin, 5-HIAA, low or } \\
\text { normal HVA. }\end{array}$ \\
\hline DYT-THAPI ${ }^{2}$ & 602629 & $8 \mathrm{p} 11.21$ & THAP1 & $\begin{array}{l}\text { Second-third } \\
\text { decade }\end{array}$ & $\begin{array}{l}\text { Early-onset mostly generalized dystonia, usually } \\
\text { involving neck and upper limbs at onset. Head or } \\
\text { upper limb tremor reported as isolated or } \\
\text { presenting sign. }\end{array}$ & & - \\
\hline $\begin{array}{l}\text { PxMD-SLC2A1 } \\
\text { (DYT9, Glut1 } \\
\text { Deficiency Syndrome) }\end{array}$ & $\begin{array}{l}601042 \\
(606777)\end{array}$ & $1 \mathrm{p} 34.2$ & $S L C 2 A 1$ & Childhood & $\begin{array}{l}\text { Paroxysmal exercise-induced dystonia with or } \\
\text { without epilepsy. Cerebellar action tremor } \\
\text { frequently reported. }\end{array}$ & & $\begin{array}{l}\text { CSF: low glucose and lactate. } \\
\text { Reduced CSF to blood glucose ratio. }\end{array}$ \\
\hline $\begin{array}{l}\text { DYT-ANO3 } \\
\text { (DYT24) }\end{array}$ & 615034 & $\begin{array}{l}\text { 11p14.3- } \\
\text { p14.2 }\end{array}$ & ANO3 & $\begin{array}{l}\text { Fourth-fifth } \\
\text { decade }\end{array}$ & $\begin{array}{l}\text { Focal or segmental dystonia. Head and/or arm } \\
\text { tremor often precedes overt dystonia. Sometimes } \\
\text { combined with myoclonic jerks. }\end{array}$ & & - \\
\hline DYT-GNAL $L^{2}$ & 615073 & $18 \mathrm{p} 11.21$ & $G N A L$ & $\begin{array}{l}\text { Childhood to } \\
\text { fourth decade }\end{array}$ & $\begin{array}{l}\text { Focal or segmental and occasionally generalized } \\
\text { dystonia. Pure dystonic tremor of the upper limbs } \\
\text { may precede or follow the onset of dystonia. }\end{array}$ & & \\
\hline \multicolumn{8}{|l|}{ Spinocerebellar ataxias ${ }^{3}$} \\
\hline $\begin{array}{l}\text { SCA-ATXN2 } \\
\text { (SCA2) }\end{array}$ & 183090 & $12 \mathrm{q} 24.12$ & $A T X N 2$ & $\begin{array}{l}\text { Third-fourth } \\
\text { decade }\end{array}$ & $\begin{array}{l}\text { Slowly progressive ataxia, slow saccades, } \\
\text { parkinsonism (including rest tremor), postural } \\
\text { tremor, sensory symptoms. }\end{array}$ & & $\begin{array}{l}\text { Brain MRI: marked cerebellar atrophy; atrophy } \\
\text { of the pons, medulla oblongata, spinal cord, } \\
\text { parietal cortex, and thalamus. } \\
\text { DaTscan: possible reduced tracer uptake. }\end{array}$ \\
\hline $\begin{array}{l}\text { SCA-ATXN3 } \\
\text { (SCA3) }\end{array}$ & 109150 & $14 \mathrm{q} 32.12$ & ATXN3 & $\begin{array}{l}\text { Third-fourth } \\
\text { decade }\end{array}$ & $\begin{array}{l}\text { Ataxia, ophthalmoplegia, parkinsonism (including } \\
\text { rest tremor), dystonia, chorea, spasticity, sensory } \\
\text { symptoms, amyotrophy. }\end{array}$ & & $\begin{array}{l}\text { Brain MRI: ponto-cerebellar atrophy. } \\
\text { DaTscan: possible reduced tracer uptake. }\end{array}$ \\
\hline $\begin{array}{l}\text { SCA-CACNA1A } \\
\text { (SCA6) }\end{array}$ & 183086 & 19p13.13 & CACNA1A ${ }^{4}$ & $\begin{array}{l}\text { Adulthood } \\
\text { (usually }>50 \\
\text { years) }\end{array}$ & $\begin{array}{l}\text { Usually pure cerebellar syndrome with ataxia and } \\
\text { postural tremor; rarely parkinsonism (including } \\
\text { rest tremor). }\end{array}$ & & $\begin{array}{l}\text { Brain MRI: moderate-severe atrophy of the } \\
\text { vermis; mild atrophy of the cerebellar } \\
\text { hemispheres; mild, diffuse atrophy of cortical } \\
\text { areas in some cases. }\end{array}$ \\
\hline $\begin{array}{l}\text { SCA-ATXN7 } \\
\text { (SCA7) }\end{array}$ & 164500 & $3 \mathrm{p} 14.1$ & ATXN7 & Adolescence & $\begin{array}{l}\text { Palatal tremor, ataxia, visual disturbances, } \\
\text { ophthalmoplegia, spasticity. }\end{array}$ & & $\begin{array}{l}\text { Brain MRI: severe pontine and cerebellar } \\
\text { atrophy. }\end{array}$ \\
\hline $\begin{array}{l}\text { SCA-PPP2R2B } \\
\text { (SCA12) }\end{array}$ & 604326 & $5 \mathrm{q} 32$ & $P P P 2 R 2 B$ & $\begin{array}{l}\text { Childhood or } \\
\text { adulthood } \\
\text { ( } 8 \text { to } 62 \text { years) }\end{array}$ & $\begin{array}{l}\text { Action tremor of the arms or head, mild or absent } \\
\text { ataxia and/or limb dysmetria, pyramidal signs. } \\
\text { Parkinsonism (including rest tremor) reported. }\end{array}$ & & $\begin{array}{l}\text { Brain MRI: cerebral and/or cerebellar } \\
\text { (vermis>hemispheres) atrophy. } \\
\text { DaTscan: reduced tracer uptake in reported } \\
\text { cases with parkinsonism. }\end{array}$ \\
\hline $\begin{array}{l}\text { SCA-ITPRI } \\
\text { (SCA15/SCA16) }\end{array}$ & 606658 & $3 \mathrm{p} 26.1$ & ITPRI & Fifth decade & $\begin{array}{l}\text { Pure cerebellar ataxia, head tremor and cognitive } \\
\text { impairment. }\end{array}$ & & $\begin{array}{l}\text { Brain MRI: cerebellar atrophy (mainly of the } \\
\text { vermis). }\end{array}$ \\
\hline
\end{tabular}




\begin{tabular}{|c|c|c|c|c|c|c|c|}
\hline SCA20 & 608687 & $11 \mathrm{q} 12.2-12.3$ & unknown & $\begin{array}{l}\text { Adulthood } \\
\text { (19-64 years) }\end{array}$ & $\begin{array}{l}\text { Dysarthria, slowly progressive ataxia, palatal } \\
\text { tremor ( } 2 / 3 \text { of cases), laryngeal dystonia, } \\
\text { hypermetric horizontal saccades, minor pyramidal } \\
\text { signs, postural tremor of the arms with or } \\
\text { without involvement of the head. }\end{array}$ & & $\begin{array}{l}\text { Brain CT/MRI: early dentate calcification, } \\
\text { sometimes along with pallidal calcification; } \\
\text { mild to moderate cerebellar atrophy; increased } \\
\text { inferior olivary T2-weighted signals in cases } \\
\text { with symptomatic palatal tremor. }\end{array}$ \\
\hline \begin{tabular}{|l|}
$\begin{array}{l}\text { SCA-FGF14 } \\
\text { (SCA27) }\end{array}$ \\
\end{tabular} & 609307 & $13 \mathrm{q} 33.1$ & $F G F 14$ & Adolescence & $\begin{array}{l}\text { Ataxia, orofacial dyskinesia, cognitive impairment, } \\
\text { hand tremor, cognitive decline. }\end{array}$ & & $\begin{array}{l}\text { Brain MRI: atrophy of the cerebellar vermis } \\
\text { and hemispheres. }\end{array}$ \\
\hline \multicolumn{8}{|l|}{ Parkinson's disease $^{5}$} \\
\hline $\begin{array}{ll}\text { PARK-SNCA } \\
\text { (PARK1/PARK4) }\end{array}$ & $\begin{array}{ll}168601 / \\
605543\end{array}$ & $4 \mathrm{q} 22.1$ & SNCA & $\begin{array}{l}\text { Usually fourth- } \\
\text { fifth decade }\end{array}$ & $\begin{array}{l}\text { Parkinsonism (including rest tremor), cognitive } \\
\text { impairment. }\end{array}$ & & DaTscan: reduced tracer uptake. \\
\hline $\begin{array}{l}\text { PARK-LRRK2 } \\
\text { (PARK8) }\end{array}$ & 607060 & $12 \mathrm{q} 12$ & $L R R K 2$ & $\begin{array}{l}\text { Usually }>50 \\
\text { years }\end{array}$ & $\begin{array}{l}\text { Slowly progressive parkinsonism (including rest } \\
\text { tremor) with good response to levodopa. }\end{array}$ & & DaTscan: reduced tracer uptake. \\
\hline \begin{tabular}{|l|}
$\begin{array}{l}\text { PARK-VPS35 } \\
\text { (PARK17) }\end{array}$ \\
\end{tabular} & 614203 & $16 \mathrm{q} 11.2$ & VPS35 & $\begin{array}{l}\text { Usually fifth- } \\
\text { sixth decade }\end{array}$ & $\begin{array}{l}\text { Parkinsonism (including rest tremor) with good } \\
\text { response to levodopa. }\end{array}$ & & DaTscan: reduced tracer uptake. \\
\hline \multicolumn{8}{|c|}{ Frontotemporal dementia } \\
\hline MAPT-FTD & 600274 & $17 \mathrm{q} 21.31$ & $M A P T$ & $\begin{array}{l}\text { Third-seventh } \\
\text { decade }\end{array}$ & $\begin{array}{l}\text { FTD, parkinsonism (including rest tremor) can } \\
\text { precede FTD, rarely MND. }\end{array}$ & $\times$ & $\begin{array}{l}\text { Brain MRI: selective frontal and/or temporal } \\
\text { lobe atrophy. } \\
\text { DaTscan: reduced tracer uptake (in cases with } \\
\text { parkinsonism). }\end{array}$ \\
\hline C9orf72-FTD & 105550 & $9 \mathrm{q} 21.2$ & C9orf72 & $\begin{array}{l}\text { Fourth-seventh } \\
\text { decade }\end{array}$ & $\begin{array}{l}\text { FTD, MND, akinetic-rigid parkinsonism, rarely } \\
\text { associated with postural or rest tremor. }\end{array}$ & $x$ & $\begin{array}{l}\text { Brain MRI: selective frontal and/or temporal } \\
\text { lobe atrophy. } \\
\text { DaTscan: reduced tracer uptake (in cases with } \\
\text { parkinsonism). }\end{array}$ \\
\hline$P R N G$-FTD & 607485 & $17 \mathrm{q} 21.31$ & $P R N G$ & $\begin{array}{l}\text { Sixth-ninth } \\
\text { decade }\end{array}$ & $\begin{array}{l}\text { FTD, parkinsonism (including rest tremor) can } \\
\text { precede FTD, rarely MND. }\end{array}$ & $x$ & $\begin{array}{l}\text { Blood tests: low progranulin. } \\
\text { CSF: low progranulin. } \\
\text { Brain MRI: selective frontal and/or temporal } \\
\text { lobe atrophy. } \\
\text { DaTscan: reduced tracer uptake (in cases with } \\
\text { parkinsonism). }\end{array}$ \\
\hline FUS-FTD & 608030 & $16 \mathrm{p} 11.2$ & $F U S$ & $\begin{array}{l}\text { Fourth-fifth } \\
\text { decade }\end{array}$ & $\begin{array}{l}\text { FTD, MND, rarely parkinsonism typically } \\
\text { associated with postural tremor. }\end{array}$ & $x$ & $\begin{array}{l}\text { Brain MRI: striking caudate atrophy; frontal } \\
\text { and/or temporal lobe atrophy. } \\
\text { DaTscan: reduced tracer uptake (in cases with } \\
\text { parkinsonism). }\end{array}$ \\
\hline TARDP-FTD & 612069 & $1 \mathrm{p} 36.22$ & TARDP & $\begin{array}{l}\text { Usually sixth } \\
\text { decade }\end{array}$ & FTD, MND, parkinsonism (including rest tremor). & $x$ & $\begin{array}{l}\text { Brain MRI: selective frontal and/or temporal } \\
\text { lobe atrophy. } \\
\text { DaTscan: reduced tracer uptake (in cases with } \\
\text { parkinsonism). }\end{array}$ \\
\hline Huntington disease & 143100 & $4 \mathrm{p} 16.3$ & $H T T$ & $\begin{array}{l}\text { Usually fourth } \\
\text { decade }\end{array}$ & $\begin{array}{l}\text { Chorea, psychiatric features, cognitive impairment. } \\
\text { Akinetic-rigid parkinsonism rather than chorea in } \\
\text { juvenile-onset forms. Isolated postural hand and } \\
\text { head tremor rarely reported as presenting sign. }\end{array}$ & $x$ & $\begin{array}{l}\text { Brain MRI: caudate atrophy. } \\
\text { DaTscan: normal tracer uptake. }\end{array}$ \\
\hline Perry syndrome & 168605 & $2 \mathrm{p} 13.1$ & DCTN1 & $\begin{array}{l}\text { Fourth-fifth } \\
\text { decade }\end{array}$ & $\begin{array}{l}\text { Parkinsonism (including rest tremor), weight loss, } \\
\text { central hypoventilation, psychiatric features (apathy, } \\
\text { hallucinations). Family history of parkinsonism and } \\
\text { sudden death. }\end{array}$ & $\times$ & DaTscan: reduced tracer uptake. \\
\hline \multicolumn{8}{|c|}{ Hereditary spastic paraplegias } \\
\hline $\begin{array}{l}\text { HSP-SPAST } \\
\text { (HSP4) }\end{array}$ & 182601 & $2 \mathrm{p} 22.3$ & $S P A S T$ & $\begin{array}{l}\text { Infancy to } \\
\text { seventh decade }\end{array}$ & $\begin{array}{l}\text { Pure (mostly) or complex HSP (cognitive } \\
\text { impairment, epilepsy, ataxia, distal amyotrophy, } \\
\text { ataxia, postural hand tremor, neuropathy) }\end{array}$ & & $\begin{array}{l}\text { Brain MRI: white matter abnormalities, } \\
\text { posterior fossa abnormalities. }\end{array}$ \\
\hline
\end{tabular}




\begin{tabular}{|c|c|c|c|c|c|c|c|}
\hline $\begin{array}{l}\text { HSP-REEPI } \\
\text { (HSP31) }\end{array}$ & 610250 & $2 \mathrm{p} 11.2$ & REEP1 & $\begin{array}{l}\text { Second to } \\
\text { seventh decade }\end{array}$ & $\begin{array}{l}\text { Complex HSP with axonal neuropathy, cerebellar } \\
\text { ataxia, cognitive impairment. Orthostatic tremor } \\
\text { reported in few cases. }\end{array}$ & & - \\
\hline $\begin{array}{l}\text { HSP-REEP2 } \\
\text { (HSP72) }\end{array}$ & 615625 & $5 q 31.2$ & REEP2 & Infancy & Spastic paraparesis, mild postural tremor. & & - \\
\hline $\begin{array}{l}\text { NBIA/CHOREA-FTL } \\
\text { (Neuroferritinopathy) }\end{array}$ & 606159 & $19 \mathrm{q} 13.33$ & $F T L$ & $\begin{array}{l}\text { Usually } \\
\text { adulthood } \\
\text { (second to sixth } \\
\text { decade) }\end{array}$ & $\begin{array}{l}\text { Progressive chorea and dystonia (mainly affecting } \\
\text { the orofacial region), palatal tremor, cognitive } \\
\text { decline, psychiatric features. }\end{array}$ & & $\begin{array}{l}\text { Blood tests: low ferritin, liver function tests } \\
\text { may be abnormal. } \\
\text { Brain MRI: iron deposition in the basal ganglia } \\
\text { and dentate; cystic degeneration of putamen } \\
\text { and globus pallidus; cortical lining sign. }\end{array}$ \\
\hline $\begin{array}{l}\text { HSP/NBIA-C19orf12 } \\
\text { (HSP43, MPAN) })^{2}\end{array}$ & 614298 & $19 q 12$ & C19orf12 & Childhood & $\begin{array}{l}\text { Spastic paraparesis, dysarthria, dystonia, } \\
\text { parkinsonism, neuropathy, psychiatric features, } \\
\text { optic atrophy. }\end{array}$ & $x$ & $\begin{array}{l}\text { Brain MRI: iron deposition in the globus } \\
\text { pallidus and substantia nigra. }\end{array}$ \\
\hline $\begin{array}{l}\text { Adult-onset Alexander } \\
\text { disease }\end{array}$ & 203450 & $17 \mathrm{q} 21.31$ & GFAP & $\begin{array}{l}\geq 13 \text { years to } \\
\text { seventh decade }\end{array}$ & $\begin{array}{l}\text { Bulbar (dysarthria, dysphonia, dysphagia) and } \\
\text { pyramidal signs, slowly progressive cerebellar } \\
\text { ataxia, palatal tremor, dysautonomia, sleep } \\
\text { disturbances, cognitive decline. Slow orthostatic } \\
\text { tremor reported in one case. }\end{array}$ & & $\begin{array}{l}\text { Brain MRI: mild to severe atrophy of the } \\
\text { medulla oblongata extending caudally to the } \\
\text { cervical spinal cord (tadpole sign) in almost } \\
90 \% \text { of cases; white matter abnormalities. } \\
\text { Neuropathology: eosinophilic inclusions } \\
\text { containing intermediate filament of GFAP } \\
\text { (Rosenthal fibers) localized in astrocyte } \\
\text { cytoplasm. }\end{array}$ \\
\hline \multirow{3}{*}{$\begin{array}{l}\text { Primary familial brain } \\
\text { calcification (Fahr } \\
\text { disease) }\end{array}$} & 213600 & $8 \mathrm{p} 11.21$ & SLC2OA2 & \multirow{3}{*}{$\begin{array}{l}\text { Third-fourth } \\
\text { decade }\end{array}$} & \multirow{3}{*}{$\begin{array}{l}\text { Progressive parkinsonism (including rest tremor), } \\
\text { dystonia, action tremor, psychiatric features, } \\
\text { headache, seizures. }\end{array}$} & & \multirow{3}{*}{$\begin{array}{l}\text { Brain CT/MRI: basal ganglia and dentate } \\
\text { calcification. }\end{array}$} \\
\hline & 615007 & $5 \mathrm{q} 32$ & PDGFRB & & & & \\
\hline & $\begin{array}{l}615483 \\
616413\end{array}$ & $\begin{array}{l}22 \mathrm{q} 13.1 \\
1 \mathrm{q} 25.3\end{array}$ & $\begin{array}{l}P D G F B \\
X P R I\end{array}$ & & & & \\
\hline $\begin{array}{l}\text { Hereditary diffuse } \\
\text { leukoencephalopathy with } \\
\text { spheroids }\end{array}$ & 221820 & $5 \mathrm{q} 32$ & CSF1R & $\begin{array}{l}\text { Usually fourth } \\
\text { decade }\end{array}$ & $\begin{array}{l}\text { Personality changes, cognitive impairment, seizures, } \\
\text { parkinsonism (including rest tremor), kinetic } \\
\text { tremor, spasticity. }\end{array}$ & $x$ & $\begin{array}{l}\text { Brain CT-MRI: brain calcification in the white } \\
\text { matter. } \\
\text { Brain MRI: cerebral white matter lesions, } \\
\text { cerebral atrophy manifesting as enlarged } \\
\text { ventricles. } \\
\text { Neuropathology: non-inflammatory myelin } \\
\text { loss, reactive astrocytosis, and axonal spheroids } \\
\text { in the white matter. }\end{array}$ \\
\hline $\begin{array}{l}\text { Charcot-Marie-Tooth } \\
\text { disease }^{7}\end{array}$ & Various & Various & $\begin{array}{l}\text { Various genes } \\
\text { (i.e. } P M P 22 \text {, } \\
M P Z, N E F L \text { ) }\end{array}$ & $\begin{array}{l}\text { Usually second } \\
\text { to third decade }\end{array}$ & $\begin{array}{l}\text { Sensory-motor neuropathy, pes cavus, peroneal } \\
\text { muscular atrophy, postural tremor. }\end{array}$ & $x$ & $\begin{array}{l}\text { NCS: MCVs ranging from demyelinating to } \\
\text { axonal. }\end{array}$ \\
\hline
\end{tabular}

Legend: 5-HIAA = 5-hydroxyindoleacetic acid; CSF = cerebrospinal fluid; CT = computed tomography; FTD = frontotemporal dementia; HSP = hereditary spastic paraparesis; $\mathrm{HVA}=$ homovanillic acid $\mathrm{MCV}=$ motor conduction velocity; $\mathrm{MND}=$ motor neuron disease; $\mathrm{MRI}=$ magnetic resonance imaging; $\mathrm{NCS}=$ nerve conduction study; $\mathrm{OMIM}^{\circledR}=$ Online Mendelian Inheritance in Man.

${ }^{\wedge}$ Tremor features are reported in bold. ${ }^{\S}$ Tremor is rarely part of the clinical spectrum. ${ }^{1}$ All autosomal dominant primary dystonias may include tremor as part of their phenotype. ${ }^{2}$ Autosomal recessive inheritance also reported. ${ }^{3}$ All autosomal dominant spinocerebellar ataxias may include tremor as part of their cerebellar 
syndrome. ${ }^{4}$ Anecdotal reports on CACNA1A-related adult-onset paroxysmal head tremor. ${ }^{5}$ All autosomal dominant forms of Parkinson's disease may include tremor as part of their phenotype. ${ }^{6}$ Autosomal recessive inheritance also reported. ${ }^{7}$ When tremor was a major feature, Charcot-Marie-Tooth disease was referred to as Roussy-Lévy syndrome in previous literature. 
Table 3. Main autosomal recessive inherited disorders which can present with tremor 


\begin{tabular}{|c|c|c|c|c|c|c|c|}
\hline Tremor disorder & OMIM $^{\circledR}$ & Chromosome & Gene & Age of onset & Main phenotype $^{\wedge}$ & $\begin{array}{l}\text { Tremor } \\
\text { as rare } \\
\text { sign }^{\S}\end{array}$ & Laboratory and imaging findings \\
\hline Wilson disease & 277900 & $13 \mathrm{q} 14.3$ & $A T P 7 B$ & $\begin{array}{l}\text { Adolescence or } \\
\text { early adulthood }\end{array}$ & $\begin{array}{l}\text { Combined dystonia with tremor (action tremor, wing- } \\
\text { beating tremor), parkinsonism (including rest tremor), } \\
\text { cerebellar signs, neuropsychiatric and cognitive features, } \\
\text { sunflower cataracts, Kayser-Fleischer ring, chronic liver } \\
\text { disease. Anecdotal reports on tongue tremor as presenting } \\
\text { sign. }\end{array}$ & & $\begin{array}{l}\text { Blood tests: low serum copper and } \\
\text { ceruloplasmin, abnormal liver } \\
\text { function. } \\
\text { Urine tests: high urinary copper. } \\
\text { MRI: high T2 signal in the basal } \\
\text { ganglia, "face of the giant panda" } \\
\text { or "double panda" signs. }\end{array}$ \\
\hline \multicolumn{8}{|l|}{ Parkinson's disease $^{1}$} \\
\hline $\begin{array}{l}\text { PARK-Parkin } \\
\text { (PARK2) }\end{array}$ & 600116 & $6 \mathrm{q} 26$ & $P R K N$ & Fourth decade & $\begin{array}{l}\text { Young-onset parkinsonism (including rest tremor) with } \\
\text { dystonic features (including dystonic tremor), mild pyramidal } \\
\text { signs. }\end{array}$ & & DaTSCAN: reduced tracer uptake. \\
\hline $\begin{array}{l}\text { PARK-PINK1 } \\
\text { (PARK6) }\end{array}$ & 605909 & $1 \mathrm{p} 36.12$ & PINK1 & $\begin{array}{l}\text { Third-fourth } \\
\text { decade }\end{array}$ & $\begin{array}{l}\text { Young-onset parkinsonism (including rest tremor) with } \\
\text { dystonic features (including dystonic tremor). Pyramidal } \\
\text { signs also reported. }\end{array}$ & & DaTSCAN: reduced tracer uptake. \\
\hline $\begin{array}{l}\text { PARK-DJ1 } \\
\text { (PARK7) }\end{array}$ & 606324 & $1 \mathrm{p} 36.23$ & $D J 1$ & $\begin{array}{l}\text { Third-fourth } \\
\text { decade }\end{array}$ & $\begin{array}{l}\text { Young-onset parkinsonism (including rest tremor), areflexia, } \\
\text { psychiatric features. }\end{array}$ & & DaTSCAN: reduced tracer uptake. \\
\hline $\begin{array}{l}\text { PARK-FBOX7 } \\
\text { (PARK15) }\end{array}$ & 260300 & $22 \mathrm{q} 12.3$ & FBOX7 & Third decade & Dystonia-parkinsonism-pyramidal syndrome. & & DaTSCAN: reduced tracer uptake. \\
\hline $\begin{array}{l}\text { PARK-SYNJ1 } \\
\text { (PARK20) }\end{array}$ & 615530 & $21 \mathrm{q} 22.11$ & SYNJ1 & Third decade & $\begin{array}{l}\text { Young-onset parkinsonism (including rest tremor), dystonia, } \\
\text { cognitive decline, seizures, eyelid apraxia. }\end{array}$ & & DaTSCAN: reduced tracer uptake. \\
\hline $\begin{array}{l}\text { DYT/PARK-SLC6A3 } \\
\text { (Dopamine transporter } \\
\text { deficiency syndrome) }\end{array}$ & 613135 & $5 \mathrm{p} 15.33$ & $S L C 6 A 3$ & Infancy & $\begin{array}{l}\text { Infantile parkinsonism-dystonia, including rest and action } \\
\text { tremor. }\end{array}$ & & $\begin{array}{l}\text { CSF: high HVA, normal neopterin, } \\
\text { biopterin, 5-HIAA. } \\
\text { DaTSCAN: absent or reduced } \\
\text { tracer uptake. }\end{array}$ \\
\hline \begin{tabular}{|l|}
$\begin{array}{l}\text { Infantile parkinsonism- } \\
\text { dystonia type } 2\end{array}$ \\
\end{tabular} & 618049 & $10 \mathrm{q} 25.3$ & $S L C 18 A 2$ & Infancy & $\begin{array}{l}\text { Severe psychomotor disability, axial hypotonia, dystonia, } \\
\text { parkinsonism (including rest tremor), oculogyric crises. }\end{array}$ & & $\begin{array}{l}\text { DaTSCAN: absent or reduced } \\
\text { tracer uptake. }\end{array}$ \\
\hline \multicolumn{8}{|l|}{ Primary dystonias $^{2}$} \\
\hline $\begin{array}{l}\text { DYT-GCHI } \\
\text { (DYT5a) }\end{array}$ & 128230 & $14 \mathrm{q} 22.2$ & GCH1 & $\begin{array}{l}\text { First-sixth } \\
\text { decade }\end{array}$ & $\begin{array}{l}\text { Progressive dopa-responsive dystonia with diurnal fluctuation. } \\
\text { Sometimes combined with parkinsonism (including rest } \\
\text { tremor) and pyramidal signs. }\end{array}$ & & $\begin{array}{l}\text { Blood tests: high post-load } \\
\text { phenylalanine. } \\
\text { CSF: low neopterin, biopterin, 5- } \\
\text { HIAA, low or normal HVA. } \\
\end{array}$ \\
\hline $\begin{array}{l}\text { DYT-TH } \\
\text { (DYT5b) }\end{array}$ & 191290 & $11 \mathrm{p} 15.5$ & $T H$ & $\begin{array}{l}\text { Infancy to } \\
\text { adolescence }\end{array}$ & $\begin{array}{l}\text { Dystonia-parkinsonism, oculogyric crisis (paroxysmal, } \\
\text { conjugate, tonic upward deviation of the eyes), autonomic } \\
\text { disturbance, tremor and myoclonus. }\end{array}$ & & $\begin{array}{l}\text { CSF: low HVA, normal neopterin, } \\
\text { biopterin, 5-HIAA. }\end{array}$ \\
\hline \multicolumn{8}{|c|}{ Autosomal recessive hereditary ataxias ${ }^{4}$} \\
\hline Friedreich ataxia & 229300 & $9 \mathrm{q} 21.11$ & $F X N$ & $\begin{array}{l}\text { First-second } \\
\text { decade (late } \\
\text { onset reported) }\end{array}$ & $\begin{array}{l}\text { Cerebellar/sensory ataxia, areflexia, muscle weakness, } \\
\text { postural or kinetic tremor, head tremor. Chorea and } \\
\text { dystonia also reported. }\end{array}$ & & $\begin{array}{l}\text { NCS: generally show MCV greater } \\
\text { than } 40 \mathrm{~m} / \mathrm{s} \text { with reduced or absent } \\
\text { SNAP with an absent } \mathrm{H} \text { reflex. } \\
\text { Brain and spine MRI: often normal } \\
\text { in early stages, atrophy of the } \\
\text { cervical spinal cord and cerebellum } \\
\text { in advanced stages. }\end{array}$ \\
\hline Ataxia telangiectasia & 208900 & $11 \mathrm{q} 22.3$ & $A T M$ & $\begin{array}{l}\text { Infancy (late } \\
\text { onset reported) }\end{array}$ & $\begin{array}{l}\text { Cerebellar ataxia, oculomotor apraxia, conjunctival } \\
\text { telangiectasias, immune defects, predisposition to malignancy. } \\
\text { Tremor reported since early stages. Parkinsonism including } \\
\text { rest tremor may be present in late stages. }\end{array}$ & & $\begin{array}{l}\text { Blood tests: high alpha-fetoprotein. } \\
\text { Brain MRI: cerebellar atrophy. }\end{array}$ \\
\hline $\begin{array}{l}\text { Ataxia with oculomotor } \\
\text { apraxia type } 1\end{array}$ & 208920 & $9 \mathrm{p} 21.1$ & APTX & $\begin{array}{l}\text { First-second } \\
\text { decade (late } \\
\text { onset reported) }\end{array}$ & $\begin{array}{l}\text { Phenotype similar to ataxia telangiectasia with sensorimotor } \\
\text { neuropathy and cognitive impairment. Chorea, dystonia, and } \\
\text { parkinsonism including rest tremor reported. }\end{array}$ & & $\begin{array}{l}\text { Blood tests: low albumin; high } \\
\text { serum cholesterol. } \\
\text { Brain MRI: cerebellar atrophy. }\end{array}$ \\
\hline $\begin{array}{l}\text { Ataxia with oculomotor } \\
\text { apraxia type } 2\end{array}$ & 606002 & $9 \mathrm{q} 34.13$ & SETX & $\begin{array}{l}\text { First-second } \\
\text { decade (late } \\
\text { onset reported) }\end{array}$ & $\begin{array}{l}\text { Phenotype similar to ataxia with oculomotor apraxia type } 1 . \\
\text { Chorea, dystonia, and parkinsonism including rest tremor } \\
\text { reported. }\end{array}$ & & $\begin{array}{l}\text { Blood tests: high alpha-fetoprotein. } \\
\text { Brain MRI: cerebellar atrophy. }\end{array}$ \\
\hline
\end{tabular}




\begin{tabular}{|c|c|c|c|c|c|c|c|}
\hline $\begin{array}{l}\text { Hereditary ataxia with } \\
\text { vitamin } E \text { deficiency }\end{array}$ & 277460 & $8 \mathrm{q} 12.3$ & $T T P A$ & $\begin{array}{l}\text { First-second } \\
\text { decade }\end{array}$ & $\begin{array}{l}\text { Friedreich-like phenotype with visual impairment or retinitis } \\
\text { pigmentosa. Dystonic head tremor reported as presenting } \\
\text { feature. }\end{array}$ & & Blood tests: low vitamin E. \\
\hline Abetalipoproteinemia & 200100 & $4 \mathrm{q} 23$ & MTTP & $\begin{array}{l}\text { First-second } \\
\text { decade }\end{array}$ & $\begin{array}{l}\text { Friedreich-like phenotype, retinitis pigmentosa, } \\
\text { hypocholesterolemia, malabsorption. }\end{array}$ & & $\begin{array}{l}\text { Blood tests: absent or extremely } \\
\text { low LDL-cholesterol, triglyceride, } \\
\text { and apolipoprotein B plasmatic } \\
\text { levels; acanthocytosis; low vitamin } \\
\text { E. }\end{array}$ \\
\hline $\begin{array}{l}\text { Cerebrotendinous } \\
\text { xanthomatosis }\end{array}$ & 213700 & $2 \mathrm{q} 35$ & CYP27A1 & $\begin{array}{l}\text { First-second } \\
\text { decade (late } \\
\text { onset reported) }\end{array}$ & $\begin{array}{l}\text { Cerebellar ataxia, cognitive impairment, juvenile cataract, } \\
\text { tendon xanthomas. Rarely parkinsonism, including rest } \\
\text { tremor. Chronic diarrhea, psychiatric symptoms, peripheral } \\
\text { neuropathy, dystonia, myoclonus, spastic paraplegia, } \\
\text { pseudobulbar palsy, seizures. }\end{array}$ & & $\begin{array}{l}\text { Blood tests: high cholestanol, } \\
\text { absent 27-OH. } \\
\text { Brain MRI: variable cerebellar } \\
\text { atrophy, cerebellar or cerebral } \\
\text { leukodystrophy. }\end{array}$ \\
\hline \multicolumn{8}{|c|}{ P } \\
\hline $\begin{array}{l}\text { NBIA/DYT-PANK2 } \\
\text { (PKAN) }\end{array}$ & 234200 & $20 \mathrm{p} 13$ & PANK2 & $\begin{array}{l}\text { Childhood (late } \\
\text { onset reported) }\end{array}$ & $\begin{array}{l}\text { Parkinsonism (including rest tremor), behavioural changes, } \\
\text { pigmentary retinopathy in } 50 \% \text { of cases. }\end{array}$ & & $\begin{array}{l}\text { Brain MRI: iron deposition in the } \\
\text { basal ganglia with "eye of the tiger" } \\
\text { sign. }\end{array}$ \\
\hline $\begin{array}{l}\text { NBIA/DYT/PARK- } \\
\text { PLA2G6 } \\
\text { (PLAN) }\end{array}$ & 256600 & $22 \mathrm{q} 13.1$ & PLA2G6 & $\begin{array}{l}\text { Childhood (late } \\
\text { onset reported) }\end{array}$ & $\begin{array}{l}\text { Parkinsonism (including rest tremor), pyramidal signs, } \\
\text { cognitive decline, cerebellar ataxia. }\end{array}$ & & $\begin{array}{l}\text { Brain MRI: usually iron deposition } \\
\text { in the globus pallidus and } \\
\text { substantia nigra; cerebellar atrophy } \\
\text { in late-onset forms. }\end{array}$ \\
\hline $\begin{array}{l}\text { HSP/NBIA-C19orf12 } \\
\text { (HSP43, MPAN) })^{3}\end{array}$ & 614298 & $19 \mathrm{q} 12$ & C19orf12 & Childhood & $\begin{array}{l}\text { Spastic paraparesis, dysarthria, dystonia, parkinsonism, } \\
\text { neuropathy, psychiatric features, optic atrophy. }\end{array}$ & & $\begin{array}{l}\text { Brain MRI: iron deposition in the } \\
\text { globus pallidus and substantia } \\
\text { nigra. }\end{array}$ \\
\hline CoPAN & 615643 & $17 \mathrm{q} 21.2$ & COASY & Childhood & $\begin{array}{l}\text { Global developmental delay, ataxia, pyramidal signs, dystonia, } \\
\text { parkinsonism. }\end{array}$ & & $\begin{array}{l}\text { Brain MRI: iron deposition in the } \\
\text { globus pallidus and substantia } \\
\text { nigra. }\end{array}$ \\
\hline $\begin{array}{l}\text { HSP/NBIA-FA2H } \\
\text { (HSP35, FAHN) }\end{array}$ & 234200 & $20 \mathrm{p} 13$ & $F A 2 H$ & $\begin{array}{l}\text { First-second } \\
\text { decade }\end{array}$ & Spastic paraparesis, ataxia, dystonia. & & $\begin{array}{l}\text { Brain MRI: iron deposition in the } \\
\text { basal ganglia (not constant), } \\
\text { pontocerebellar atrophy, white } \\
\text { matter hyperintensities. }\end{array}$ \\
\hline $\begin{array}{l}\text { Kufor-Rakeb disease } \\
\text { (PARK-ATP13A2) }\end{array}$ & 606693 & $1 \mathrm{p} 36.13$ & ATP13A2 & $\begin{array}{l}\text { Second-third } \\
\text { decade }\end{array}$ & $\begin{array}{l}\text { Early-onset levodopa-responsive parkinsonism (including rest } \\
\text { tremor), pyramidal features, supranuclear upgaze palsy, } \\
\text { cognitive impairment. }\end{array}$ & & $\begin{array}{l}\text { Brain MRI: generalized atrophy, } \\
\text { putaminal and caudate iron } \\
\text { deposition. }\end{array}$ \\
\hline $\begin{array}{l}\text { NBIA/DYT/PARK-CP } \\
\text { (Aceruloplasminemia) }\end{array}$ & 604290 & $3 q 24-q 25$ & $C P$ & $\begin{array}{l}\text { Middle } \\
\text { adulthood }\end{array}$ & $\begin{array}{l}\text { Retinal degeneration, diabetes, neurological features (dystonia, } \\
\text { parkinsonism including rest tremor, ataxia). }\end{array}$ & & $\begin{array}{l}\text { Blood tests: absent serum } \\
\text { ceruloplasmin, high serum ferritin. } \\
\text { Brain MRI: iron deposition in the } \\
\text { basal ganglia and dentate. }\end{array}$ \\
\hline Glutaric aciduria type 1 & 231670 & $19 \mathrm{p} 13.13$ & $G C D H$ & $\begin{array}{l}\text { Infancy or } \\
\text { adulthood }\end{array}$ & $\begin{array}{l}\text { Infancy: acute systemic complications, generalized dystonia. } \\
\text { Later in life: action tremor of the arms and head, dystonia, } \\
\text { cerebellar and pyramidal signs, premature ovarian failure in } \\
\text { females. }\end{array}$ & & $\begin{array}{l}\text { Routine newborn screening in some } \\
\text { countries. }\end{array}$ \\
\hline Classic galactosemia & 230400 & $9 \mathrm{p} 13.3$ & GALT & $\begin{array}{l}\text { Neonatal period } \\
\text { to adulthood }\end{array}$ & $\begin{array}{l}\text { Infancy: acute encephalopathic crises precipitated by febrile } \\
\text { episodes. Later in life: bibrachial dystonic tremor along with } \\
\text { orofacial dyskinesias. }\end{array}$ & & $\begin{array}{l}\text { Routine newborn screening in some } \\
\text { countries. }\end{array}$ \\
\hline \multicolumn{8}{|l|}{ Manganese transportopathies } \\
\hline SLC30A10 deficiency & 613280 & $1 \mathrm{q} 41$ & SLC30A10 & $\begin{array}{l}\text { Childhood or } \\
\text { adulthood }\end{array}$ & $\begin{array}{l}\text { Liver disease, polycythemia. Childhood: limb dystonia, } \\
\text { dysarthria, fine tremor, bradykinesia, spastic paraparesis. } \\
\text { Adulthood: parkinsonism, including rest tremor. }\end{array}$ & $x$ & $\begin{array}{l}\text { Blood tests: abnormal liver } \\
\text { function, polycythemia. } \\
\text { Brain MRI: T1 hyperintensities of } \\
\text { the basal ganglia. }\end{array}$ \\
\hline
\end{tabular}




\begin{tabular}{|c|c|c|c|c|c|c|c|}
\hline SLC39A14 deficiency & 617013 & $8 \mathrm{p} 21.3$ & SLC39A14 & Infancy & $\begin{array}{l}\text { Loss of developmental milestones, dystonia, bulbar signs, } \\
\text { spasticity. }\end{array}$ & $x$ & $\begin{array}{l}\text { Brain MRI: T1 hyperintensities of } \\
\text { the basal ganglia. }\end{array}$ \\
\hline \multicolumn{8}{|c|}{ Hereditary spastic paraplegias } \\
\hline $\begin{array}{l}\text { HSP-KIAA1840 } \\
\text { (HSP11) }\end{array}$ & 604360 & $15 \mathrm{q} 21.1$ & KIAA1840 & $\begin{array}{l}\text { Infancy or } \\
\text { adolescence (late } \\
\text { onset reported) }\end{array}$ & $\begin{array}{l}\text { Complex HSP. Cerebellar signs, polyneuropathy, seizures, } \\
\text { cognitive impairment, abnormal eye signs, amyotrophy, } \\
\text { parkinsonism (including rest tremor), maculopathy, action } \\
\text { tremor, mental retardation, upper limb weakness. }\end{array}$ & & $\begin{array}{l}\text { Brain MRI: white matter } \\
\text { abnormalities, cerebellar atrophy, } \\
\text { thin corpus callosum, "ears of the } \\
\text { lynx" sign. }\end{array}$ \\
\hline $\begin{array}{l}\text { HSP-DSTYK } \\
\text { (HSP23) }\end{array}$ & 270750 & $1 \mathrm{q} 32.1$ & DSTYK & Childhood & $\begin{array}{l}\text { Cognitive impairment, Lison syndrome, pigmentary skin } \\
\text { abnormalities, facial and skeletal dysmorphism, tremor. }\end{array}$ & & 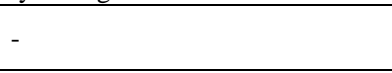 \\
\hline $\begin{array}{l}\text { HSP-GJC2 } \\
\text { (HSP44) }\end{array}$ & 613206 & $1 \mathrm{q} 42.13$ & GJC2 & Adulthood & $\begin{array}{l}\text { Pes cavus and a late onset slowly progressive paraplegia, } \\
\text { dysarthria, loss of finger dexterity, dysmetria, intention } \\
\text { tremor, scoliosis, upper limb involvement, and cognitive } \\
\text { impairment. }\end{array}$ & & $\begin{array}{l}\text { Brain MRI: hypomyelinating } \\
\text { leukodystrophy and TCC in all } \\
\text { three } \\
\text { patients }\end{array}$ \\
\hline $\begin{array}{l}\text { HSP-PGAPI } \\
\text { (HSP67) }\end{array}$ & N.A. & $2 q 33.1$ & $P G A P 1$ & Infancy & $\begin{array}{l}\text { Severe developmental delay, Distended abdomen, borderline } \\
\text { intelligence, ACC, vermis hypoplasia, defective myelination }\end{array}$ & $x$ & $\begin{array}{l}\text { Brain MRI: agenesis of corpus } \\
\text { callosum, hypomyelination. }\end{array}$ \\
\hline $\begin{array}{l}\text { HSP-REEP2 } \\
(\text { HSP72) }\end{array}$ & 615625 & $5 q 31.2$ & REEP2 & Early childhood & Spastic paraparesis, mild postural tremor. & & (n) \\
\hline $\begin{array}{l}\text { Charcot-Marie-Tooth } \\
\text { disease }^{6}\end{array}$ & Various & Various & $\begin{array}{l}\text { Various genes } \\
\text { (i.e. } M F N 2 \text {, } \\
N E F L \text { ) }\end{array}$ & $\begin{array}{l}\text { Usually second } \\
\text { to third decade }\end{array}$ & $\begin{array}{l}\text { Sensory-motor neuropathy, pes cavus, peroneal muscular } \\
\text { atrophy, postural tremor. }\end{array}$ & $x$ & $\begin{array}{l}\text { NCS: MCVs ranging from } \\
\text { demyelinating to axonal. }\end{array}$ \\
\hline
\end{tabular}

Legend: 5-HIAA = 5-hydroxyindoleacetic acid; CSF = cerebrospinal fluid; CT = computed tomography; HSP = hereditary spastic paraparesis; HVA = homovanillic acid $\mathrm{MCV}=$ motor conduction velocity; $\mathrm{MND}=$ motor neuron disease; $\mathrm{MRI}=$ magnetic resonance imaging; $\mathrm{N} . \mathrm{A} .=$ not available; $\mathrm{NBIA}=$ neurodegeneration with brain iron accumulation; NCS = nerve conduction study; $\mathrm{OMIM}^{\circledR}=$ Online Mendelian Inheritance in Man; SNAP = sensory nerve action potential.

${ }^{\wedge}$ Tremor features are reported in bold. ${ }^{\S}$ Tremor is rarely part of the clinical spectrum. ${ }^{1}$ All autosomal recessive forms of Parkinson's disease may include tremor as part of their phenotype. ${ }^{2}$ All autosomal recessive primary dystonias may include tremor as part of their phenotype. ${ }^{3}$ Autosomal dominant inheritance also reported. ${ }^{4}$ All autosomal recessive spinocerebellar ataxias may include tremor as part of their cerebellar syndrome. ${ }^{5}$ Autosomal dominant inheritance also reported. ${ }^{6}$ When tremor was a major feature, Charcot-Marie-Tooth disease was referred to as Roussy-Lévy syndrome in previous literature. 
Table 4. Main X-linked inherited disorders which can present with tremor

\begin{tabular}{|c|c|c|c|c|c|c|c|}
\hline Tremor disorder & OMIM $^{\circledR}$ & Chromosome & Gene & Age of onset & Main phenotype ${ }^{\wedge}$ & $\begin{array}{l}\text { Tremor } \\
\text { as rare } \\
\text { sign }^{\S}\end{array}$ & Laboratory and imaging findings \\
\hline $\begin{array}{l}\text { Fragile } X \text { tremor/ataxia } \\
\text { syndrome }\end{array}$ & 300623 & $\mathrm{Xq} 27.3$ & FMRI & $\begin{array}{l}\text { Middle-late } \\
\text { adulthood } \\
\text { (usually }>50 \text { years) }\end{array}$ & $\begin{array}{l}\text { Intention or action tremor, cerebellar ataxia, cognitive } \\
\text { decline, neuropathy, vestibular and autonomic dysfunction. } \\
\text { Postural and rest tremor also reported. Family history: } \\
\text { intellectual disability in male relatives; premature ovarian } \\
\text { failure in female relatives. }\end{array}$ & & $\begin{array}{l}\text { Brain MRI: middle cerebellar peduncle } \\
\text { sign, moderate to severe brain atrophy, } \\
\text { cerebral and cerebellar white matter } \\
\text { abnormalities. }\end{array}$ \\
\hline $\begin{array}{l}\text { DYT-TAF1 } \\
\text { (DYT3, Lubag } \\
\text { syndrome) }\end{array}$ & 314250 & $\mathrm{Xq} 13.1$ & $T A F 1$ & Third-fourth decade & Dystonia, parkinsonism, including rest tremor. & & $\begin{array}{l}\text { Brain MRI: hyperintense putaminal rim, } \\
\text { then atrophy of the caudate head or } \\
\text { putamen. }\end{array}$ \\
\hline Waisman syndrome & 311510 & $\mathrm{Xq} 28$ & $R A B 39 B$ & $\begin{array}{l}\text { Early adulthood } \\
(<45 \text { years })\end{array}$ & $\begin{array}{l}\text { Intellectual disability, early-onset parkinsonism, including } \\
\text { rest tremor. }\end{array}$ & & P \\
\hline Adrenoleukodystrophy & 300100 & $\mathrm{Xq} 28$ & $A B C D 1$ & Childhood/Adulthood & $\begin{array}{l}\text { Spastic paraparesis, adrenal insufficiency, bladder and bowel } \\
\text { dysfunction. }\end{array}$ & & $\begin{array}{l}\text { Blood tests: high very long chain fatty } \\
\text { acids, high adrenocorticotropic hormone. } \\
\text { Brain MRI: white matter abnormalities. }\end{array}$ \\
\hline Rett syndrome & 312750 & $\mathrm{Xq} 28$ & MECP2 & $\begin{array}{l}\text { Infancy } \\
\text { (6-18 months) }\end{array}$ & $\begin{array}{l}\text { Occurs almost exclusively in females. Developmental arrest } \\
\text { and regression, poor sociability, lack of communication, } \\
\text { irritability, anxiety, dystonia, tremor, respiratory pauses, } \\
\text { loss of hand skills, deceleration of head growth, progressive } \\
\text { motor deterioration and stereotypies. }\end{array}$ & & (1) \\
\hline Lesch-Nyhan syndrome & 300322 & $\mathrm{Xq} 26.2-26.3$ & $H P R T 1$ & $\begin{array}{l}\text { Infancy } \\
\text { (3-6 months) }\end{array}$ & $\begin{array}{l}\text { Dystonia (including dystonic tremor), choreoathetosis, } \\
\text { ballismus, cognitive and attentional deficits, self-injurious } \\
\text { behaviors. }\end{array}$ & & Blood tests: high uric acid. \\
\hline $\begin{array}{l}\text { Spinal and bulbar } \\
\text { muscle atrophy } \\
\text { (Kennedy disease) }\end{array}$ & 313200 & $\mathrm{Xq} 12$ & $A R$ & $\begin{array}{l}\text { Third-fifth decade } \\
\text { (may appear earlier) }\end{array}$ & $\begin{array}{l}\text { Lower motor neuron disease (muscle weakness and atrophy, } \\
\text { fasciculations, decreased or absent tendon reflexes) } \\
\text { involving the limbs and bulbar region (dysarthria and } \\
\text { dysphagia); cramps; sensory neuropathy; gynecomastia; } \\
\text { sexual dysfunction (testicular atrophy, decreased fertility, } \\
\text { erectile dysfunction); partial metabolic syndrome (diabetes } \\
\text { mellitus, non-alcoholic fatty liver disease). High-frequency } \\
\text { postural tremor of the hands, which may precede the } \\
\text { onset of muscle weakness. Postural leg tremor also } \\
\text { reported. A minority of female carriers have mild symptoms } \\
\text { (cramps; tremor). }\end{array}$ & & $\begin{array}{l}\text { Blood tests: elevated CPK; hyperglycemia; } \\
\text { hyperlipidemia. } \\
\text { EMG/NCS: signs of acute and chronic } \\
\text { diffuse denervation; low SNAP amplitudes. }\end{array}$ \\
\hline $\begin{array}{l}\text { Pelizaeus-Merzbacher } \\
\text { disease }\end{array}$ & 312080 & $\mathrm{Xq} 22.2$ & $P L P 1$ & Early childhood & $\begin{array}{l}\text { Pyramidal dysfunction, cerebellar ataxia, head tremor, } \\
\text { progressive nystagmus, psychomotor delay. }\end{array}$ & $x$ & Brain MRI: hypomyelination. \\
\hline $\begin{array}{l}\text { NBIA/PARK-WDR45 } \\
\text { (BPAN) }\end{array}$ & 300894 & Xp11.23 & WDR45 & Early childhood & $\begin{array}{l}\text { First stage (early childhood): developmental delay, } \\
\text { intellectual disability, epilepsy, stereotypies, dysfunctional } \\
\text { sleep, ocular defects. Second stage (adolescence or early } \\
\text { adulthood): rapidly progressive parkinsonism and cognitive } \\
\text { decline. Rest tremor may be part of parkinsonism. Both } \\
\text { males and females present with a similar phenotype. }\end{array}$ & & $\begin{array}{l}\text { Brain MRI: in the first stage normal or } \\
\text { nonspecific brain atrophy; in the second } \\
\text { stage iron accumulation in the substantia } \\
\text { nigra and globus pallidus (hypointense on } \\
\text { T2); on T1 hyperintense "halo" surrounding } \\
\text { a thin line of hypointensity in the substantia } \\
\text { nigra and cerebral peduncles. }\end{array}$ \\
\hline Cabezas syndrome & 300354 & $\mathrm{Xq} 24$ & $C U L A B$ & Infancy & $\begin{array}{l}\text { Intellectual disability; speech delay; short stature; } \\
\text { cervicodorsal kyphosis; hyperextensible joints; truncal } \\
\text { obesity; abnormal gait; prominent lower lip; hypogonadism; }\end{array}$ & & $\begin{array}{l}\text { Brain MRI: cortical dysplasia; } \\
\text { ventriculomegaly; loss of cerebral white } \\
\text { matter; thin corpus callosum. }\end{array}$ \\
\hline
\end{tabular}




\begin{tabular}{|c|c|c|c|c|c|c|c|}
\hline & & & & & $\begin{array}{l}\text { muscle wasting in the lower legs; small feet. Constant fine } \\
\text { tremor in the upper extremities with intention } \\
\text { component. Female carriers are normal or minimally } \\
\text { affected. }\end{array}$ & & \\
\hline $\operatorname{CMTX}(G J B 2)^{1}$ & 302800 & $\mathrm{Xq} 13.1$ & $\begin{array}{l}\text { GJB2 } \\
(90 \% \\
\text { CMTX) }\end{array}$ & $\begin{array}{l}\text { Second to third } \\
\text { decade }\end{array}$ & $\begin{array}{l}\text { Males more severely affected than females. Sensory-motor } \\
\text { neuropathy. Postural tremor reported. }\end{array}$ & $x$ & $\begin{array}{l}\text { NCS: MCV ranging from demyelinating to } \\
\text { axonal. }\end{array}$ \\
\hline
\end{tabular}

Legend: $\mathrm{EMG}=$ electromyography; $\mathrm{MCV}=$ motor conduction velocity; $\mathrm{MRI}=$ magnetic resonance imaging; $\mathrm{NCS}=$ nerve conduction study; OMIM ${ }^{\circledR}=$ Online Mendelian Inheritance in Man.

${ }^{\wedge}$ Tremor features are reported in bold. ${ }^{\S}$ Tremor is rarely part of the clinical spectrum. ${ }^{1}$ When tremor was a major feature, CMT has been referred to as RoussyLévy syndrome in previous literature. 
Table 5. Main chromosomal abnormalities which may include tremor in their clinical picture

\begin{tabular}{|c|c|c|c|c|}
\hline Tremor disorder & Karyotype & Age of symptom onset & Main phenotype $^{\wedge}$ & Laboratory and imaging findings \\
\hline \multicolumn{5}{|c|}{ Quantitative chromosomal abnormalities } \\
\hline Klinefelter syndrome & $47, \mathrm{XXY}$ & $\begin{array}{l}\text { Puberty. } \\
\text { Tremor usually begins } \\
\text { during childhood, but may } \\
\text { appear later in life. }\end{array}$ & $\begin{array}{l}\text { Intention tremor of the upper extremities, sometimes } \\
\text { associated with head, voice, and leg tremor, tall stature, arm } \\
\text { span exceeding height, hypergonadotropic } \\
\text { hypogonadism/androgen deficiency (infertility due to } \\
\text { azoospermia, painless bilateral gynecomastia, decreased libido } \\
\text { and potency, very low testicular volume and firm consistency } \\
\text { of the testes). }\end{array}$ & $\begin{array}{l}\text { Blood tests: hypergonadotropic hypogonadism/androgen deficiency. } \\
\text { Spermiogram: azoospermia. }\end{array}$ \\
\hline Jacobs syndrome & $47, \mathrm{XYY}$ & First decade & $\begin{array}{l}\text { Intention tremor of the arms, either unilateral or bilateral, } \\
\text { sometimes with postural and/or mild resting tremor of the } \\
\text { arms as well as head tremor. Tall stature starting from } \\
\text { childhood, a tendency toward central adiposity, macrocephaly, } \\
\text { macro-orchidism starting in early puberty, hypotonia, } \\
\text { clinodactyly, and hypertelorism. Asthma, dental problems, } \\
\text { and seizures are more frequent than in the pediatric population. } \\
\text { Attention deficit or autistic spectrum disorder. Developmental } \\
\text { delays and language disorders are common. }\end{array}$ & - \\
\hline $\begin{array}{l}\text { Other supernumerary } \\
\text { X or Y syndromes }\end{array}$ & $48, \mathrm{XXYY}$ & First decade & $\begin{array}{l}\text { Postural and/or action tremor of the upper limbs, tall } \\
\text { stature, micro-orchidism, and hypergonadotropic } \\
\text { hypogonadism, dysmorphic features (long face, epicanthal } \\
\text { folds, poor dentition), pes planus, allergies, asthma, attention } \\
\text { deficit disorder, and learning difficulties. }\end{array}$ & $\begin{array}{l}\text { Blood tests: hypergonadotropic hypogonadism/androgen deficiency. } \\
\text { Spermiogram: azoospermia. }\end{array}$ \\
\hline \multicolumn{5}{|c|}{ Qualitative chromosomal abnormalities } \\
\hline $\begin{array}{l}\text { Chromosome 22q11.2 } \\
\text { deletion syndrome }\end{array}$ & & First decade & $\begin{array}{l}\text { Various combinations of congenital heart defects (particularly } \\
\text { conotruncal malformation), palatal abnormalities, facial } \\
\text { dysmorphic features, development delays and learning } \\
\text { difficulties, immune deficiency, endocrine dysfunctions, } \\
\text { hypocalcemia. Less frequent manifestations include } \\
\text { psychiatric illness (psychosis, schizophrenia, attention deficit } \\
\text { disorder, anxiety), skeletal malformations, esophageal } \\
\text { dysmotility and structural gastrointestinal anomalies, renal } \\
\text { anomalies, seizures (idiopathic or associated with } \\
\text { hypocalcemia), ophthalmologic abnormalities, and } \\
\text { reproductive issues, parkinsonism (including rest tremor). }\end{array}$ & DaTscan: reduced tracer uptake. \\
\hline
\end{tabular}

${ }^{\wedge}$ Tremor features are reported in bold. 


\section{Figure 1. Phenotype-to-genotype algorithm for genetic tremor syndromes}

Overview: main tremor disorders discussed in this review arranged according to their syndromic classification and mode of inheritance. *Rarer presentations are mentioned in small print. The underlying genes are given in italic. 
GENETIC TREMOR DISORDERS

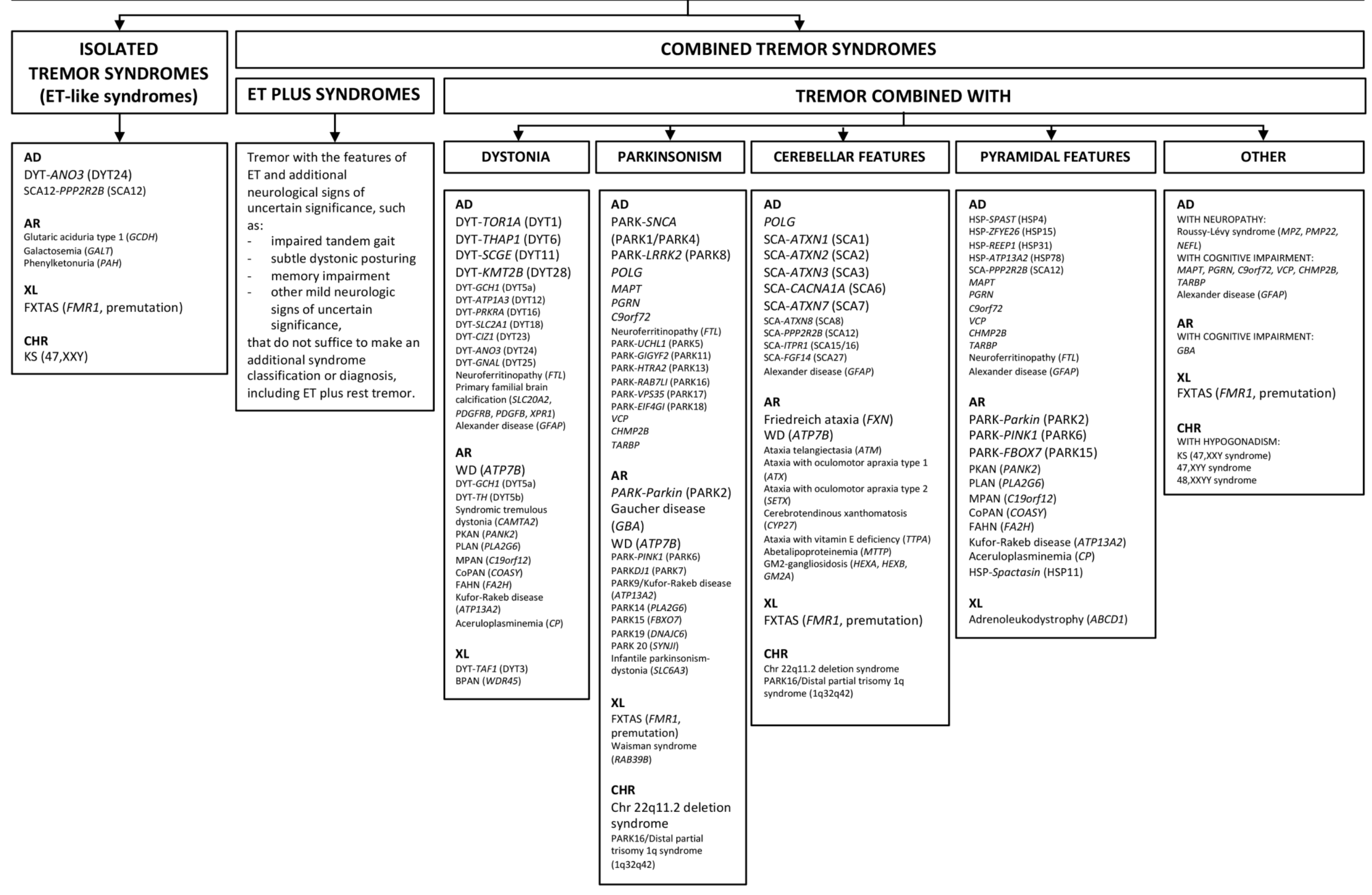


Legend: $\mathrm{AD}=$ autosomal dominant; $\mathrm{AR}=$ autosomal recessive; $\mathrm{XL}=\mathrm{X}$-linked; $\mathrm{CHR}=$ chromosomal abnormality. 
Video Legend

Video 1. Segment 1: tremor in a case of SCA-PPP2R2B (SCA12). Segment 2: tremor in a case of fragile X tremor-ataxia syndrome (FXTAS). 


\section{Supplementary File Legend}

Supplementary Table. Video collection of some tremor disorders reviewed in this article.

Previously unpublished videos (Video) or references to previously published videos (Supplementary Table) are provided.

\begin{tabular}{|l|l|}
\hline Tremor disorder & Reference to Video \\
\hline $\begin{array}{l}\text { DYT-TOR1A } \\
\text { (DYT1) }\end{array}$ & {$[\mathrm{S} 1]$} \\
\hline $\begin{array}{l}\text { DYT-ANO3 } \\
\text { (DYT24) }\end{array}$ & {$[\mathrm{S} 2]$} \\
\hline $\begin{array}{l}\text { SCA-PPP2R2B } \\
\text { (SCA12) }\end{array}$ & Video, Segment 1 \\
\hline $\begin{array}{l}\text { HSP-REEP1 } \\
\text { (HSP31) }\end{array}$ & {$[\mathrm{S} 3]$} \\
\hline Adult-onset Alexander disease & {$[\mathrm{S} 4]$} \\
\hline Wilson disease & {$[\mathrm{S} 5]$} \\
\hline Glutaric aciduria type 1 & {$[\mathrm{S} 6]$} \\
\hline Classic galactosemia & {$[\mathrm{S} 7]$} \\
\hline Fragile X tremor/ataxia syndrome & Video, Segment 2 \\
\hline $\begin{array}{l}\text { Spinal and bulbar muscle atrophy } \\
\text { (Kennedy disease) }\end{array}$ & {$[\mathrm{S} 8]$} \\
\hline Klinefelter syndrome & {$[\mathrm{S} 9]$} \\
\hline Jacobs syndrome & {$[\mathrm{S} 10]$} \\
\hline 48,XXYY syndrome & {$[\mathrm{S} 11]$} \\
\hline
\end{tabular}

\section{Supplementary References}

[S1] F. Magrinelli, R. Bacchin, M. Tinazzi, M. Gambarin, Twelve-year follow-up of a large Italian family with atypical phenotypes of DYT1-dystonia, Mov. Disord. Clin. Pract. 30 (2018): 166-170.

[S2] M. Stamelou, G. Charlesworth, C. Cordivari, S.A. Schneider, G. Kägi, U.M. Sheerin, I. Rubio-Agusti, A. Batla, H. Houlden, N.W. Wood, K.P. Bhatia, The phenotypic spectrum of DYT24 due to ANO3 mutations, Mov. Disord. 29 (2014): 928-934. 
[S3] M. Picillo, R. Erro, R.P. Munhoz, A. Fasano, When shaking during standing points to hereditary spastic paraplegias, Parkinsonism Relat. Disord. 46 (2018): 92-94.

[S4] J.R. Sebesto, J.A. van Gerpen, Teaching Video NeuroImages: Palatal tremor in adult-onset Alexander disease, Neurology 86 (2016): e252.

[S5] R. Mahajan, U. Zachariah, Images in clinical medicine. Wing-beating tremor, N. Engl. J. Med. 371 (2014): e1.

[S6] E. Fernandez-Alvarez, A. Garcia-Cazoria, A. Sans, C. Boix, M.A. Vilaseca, C. Busquets, A. Ribes, Hand tremor and orofacial dyskinesia: clinical manifestations of glutaric aciduria type I in a young girl, Mov. Disord. 18 (2003): 1076-1079.

[S7] I. Rubio-Agusti, M. Carecchio, K.P. Bhatia, M. Kojovic, I. Parees, H.S. Chandrashekar, E.J. Footitt, D. Burke, M.J. Edwards, R.H. Lachmann, E. Murphy, Movement disorders in adult patients with classical galactosemia, Mov. Disord. 28 (2013): 804-810.

[S8] I. Aicua, O. Verhagen, N. Arenaza, E. Cubo, Head and arm tremor in X-linked spinal and bulbar muscular atrophy, Tremor Other Hyperkinet. Mov. (NY) 4 (2014): 265.

[S9] M. Koegl-Wallner, P. Katschnig-Winter, T. Pendl, B. Melisch, M. Trummer, E. Holl, U. Werner, R. Schmidt, P. Schwingenschuh, Tremor associated with Klinefelter syndrome--a case series and review of the literature, Parkinsonism Relat. Disord. 20 (2014): 323-327.

[S10] J.L. Davis, J.A. Kurek, J.C. Morgan, K.D. Sethi, Tremor and dystonia in Jacob's Syndrome (47,XYY), Mov. Disord. Clin. Pract. 7 (2019): 107-108. 
[S11] N. Tartaglia, M. Borodyanskaya, D.A. Hall, Tremor in 48,XXYY syndrome, Mov. Disord. 24 (2009): 2001-2007. 